\title{
An overview of artificial intelligence applications for power electronics
}

This paper was downloaded from TechRxiv (https://www.techrxiv.org).

\section{LICENSE}

CC BY 4.0

SUBMISSION DATE / POSTED DATE

04-06-2020 / 01-10-2020

CITATION

Zhao, Shuai; Blaabjerg, Frede; Wang, Huai (2020): An overview of artificial intelligence applications for power electronics. TechRxiv. Preprint. https://doi.org/10.36227/techrxiv.12431081.v2

DOI

10.36227/techrxiv.12431081.v2 


\title{
An Overview of Artificial Intelligence Applications for Power Electronics
}

\author{
Shuai Zhao, Member, IEEE, Frede Blaabjerg, Fellow, IEEE, and Huai Wang, Senior Member, IEEE
}

\begin{abstract}
This paper gives an overview of the Artificial Intelligence (AI) applications for power electronic systems. The three distinctive life-cycle phases, design, control, and maintenance are correlated with one or more tasks to be addressed by AI, including optimization, classification, regression, and data structure exploration. The applications of four categories of AI are discussed, which are expert system, fuzzy logic, metaheuristic method, and machine learning. More than 500 publications have been reviewed to identify the common understandings, practical implementation challenges, and research opportunities in the application of AI for power electronics. This paper is accompanied by an Excel file listing the relevant publications for statistical analytics.
\end{abstract}

Index Terms-Artificial intelligence, design, intelligent controller, predictive maintenance, power electronic systems, prognostics and health management

\section{INTRODUCTION}

$\mathbf{N}$ OWADAYS artificial intelligence (AI) is expanding rapidly and is one of the most salient research areas during the last several decades [1], [2]. The aim of AI is to facilitate systems with intelligence that is capable of human-like learning and reasoning. It possesses tremendous advantages and has been successfully applied in numerous industrial areas including image classification, speech recognition, autonomous cars, computer vision, etc. With immense potentials, power electronics benefit from the development of AI. There are various applications, including design optimization of power module heatsink [3], intelligent controller for multi-color light-emitting diode (LED) [4], maximum power point tracking (MPPT) control for wind energy conversion systems [5], [6], anomaly detection for inverter [7], remaining useful life (RUL) prediction for supercapacitors [8], etc. By implementing AI, power electronic systems are embedded with capabilities of self-awareness and self-adaptability, and therefore the system autonomy can be improved.

Meanwhile, the rapid development of data science, including sensor technology, internet-of-things (IoT), edge computing, digital twin [9], and big data analytics [10], [11], provides a wide variety of data for power electronic systems throughout different phases of its life-cycle. The increasing volume of data enables immense opportunities and lays a

This work was supported by the Innovation Fund Denmark through the project of Advanced Power Electronic Technology and Tools (APETT) and the Villum Foundation through the project of Light-AI for Congitive Power Electronics.

Shuai Zhao, Frede Blaabjerg, and Huai Wang are with the Department of Energy Technology, Aalborg University, Aalborg 9220, Denmark. (Email: szh@et.aau.dk; fbl@et.aau.dk; hwa@et.aau.dk) (Corresponding author: Huai Wang). solid foundation for the AI in power electronics. AI is able to exploit data to improve product competitiveness by global design optimization, intelligent control, system health status estimation, etc. As a result, the research in power electronics can be conducted from a data-driven perspective, which is beneficial especially to complex and challenging cases.

Due to the specific challenges and characteristics of power electronic systems, e.g., high tuning speed in control, high sensitivity in condition monitoring for aging detection, etc., the implementation of $\mathrm{AI}$ in power electronics has its own features that are different from other engineering areas, e.g., image classification. Therefore, there is a pressing need for an overview of AI in power electronics to expedite synergy research and interdisciplinary applications. Based on literature review, in this paper the applications of $\mathrm{AI}$ in power electronics are categorized into three aspects, i.e., design, control, and maintenance.

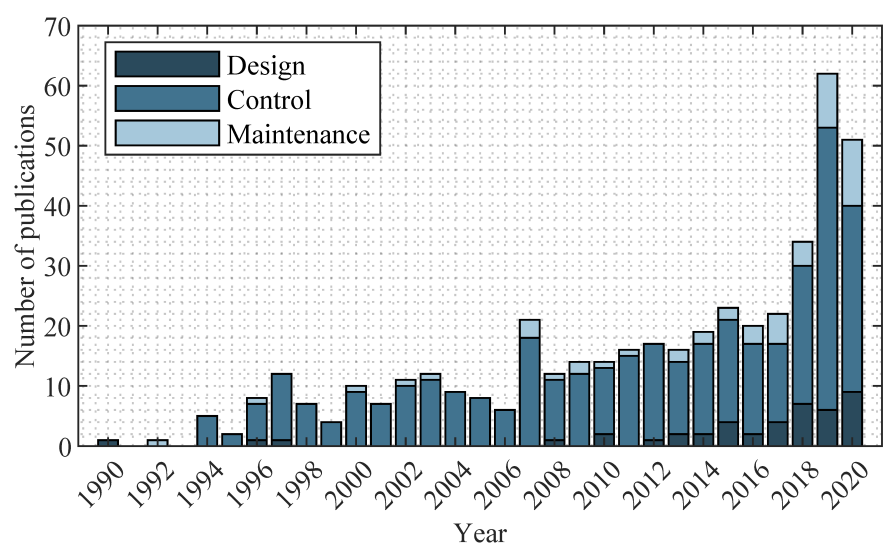

Fig. 1. Annual number of publications of AI in power electronics since 1990.

Fig. 1 shows the annual number of publications related to AI for power electronics since 1990. The statistical data are based on searching the IEEE Xplore from the journals IEEE Transactions on Power Electronics, IEEE Journal of Emerging and Selected Topics in Power Electronics, IEEE Transactions on Industrial Electronics, IEEE Transactions on Industrial Informatics, and IEEE Transactions on Industry Applications. The data of 2020 are up to May 2020. As a result, a total of 444 relevant journal papers are identified, which can be found in the supplemental Excel file. It can be seen that the implementations of AI have been drastically increased and experienced a spectacular dynamism over the last few years. The number of publications for control is continuously increasing and it is the most active research area. Since 2007, there is an increase regarding the design and maintenance 
applications, and such trends are more evident in the last two years.

It is found that several existing reviews in the literature are related to this topic. In [12], the metaheuristic methods for stochastic optimization for power quality and waveform, circuit design, and control tuning are reviewed. It focuses on the optimization tasks only. The details of neural network (NN) in industrial applications are presented in [13] with the design of network structure, training methods, and application considerations. It covers a broad scope of engineering applications beyond power electronics. In [14], a comprehensive review is given on the applications of $\mathrm{NN}$ in power electronics. Several specific examples of control and system identification are detailed. Nevertheless, other AI techniques, such as fuzzy logic, metaheuristic methods, etc., have not been discussed. Although these techniques are further discussed in [15], it emphasizes on illustrative examples while an in-depth analysis of AI algorithms is not provided. In [16], an intensive discussion of metaheuristic methods for MPPT in photovoltaic (PV) systems is presented. In [17], the AI techniques applied to PV systems are reviewed, which focuses on the specific PV applications only.

Maintenance [18] in power electronics is a topic that includes reliability, condition monitoring, remaining useful life prediction, etc. Several review papers in the last decade can be found in [19]-[22]. In [19], a state-of-the-art analysis of the condition monitoring and fault detection in power electronics is presented. However, it only includes a very limited AI-based fault detection methods. In [20], a review of condition monitoring techniques for capacitors in power electronic converters is presented. It includes only the AIbased parameter identification methods. In [21], the methods in Prognostics and Health Management (PHM) of information and electronics-rich systems are summarized. This paper only discusses the category of AI algorithms in the PHM area, while there is no algorithm detail or comparative analysis. In [22], machine learning methods applied in reliability management of energy systems are summarized. It focuses on the machine learning method and the maintenance task only. A tutorial [23] regarding "Artificial Intelligence Applications to Power Electronics" is presented on the 2019 IEEE Energy Conversion Congress and Exposition. It serves as an introductory level presentation. Nevertheless, the desirable details of the AI algorithms and their comparisons are not available.

As a result, it lacks a comprehensive review of the AI algorithms and applications for power electronics. From a lifecycle perspective, this paper aims to fill this gap and comprehensively review the published research in power electronics using AI techniques, which needs a systematic consolidation. The contributions of this paper include

1) The AI algorithms in power electronics are systematically investigated from a life-cycle perspective, where the relationships of the relevant AI algorithms, their essential functions, and the relevant applications are identified.

2) A timeline map is provided to illustrate the milestones of AI algorithms and power electronic applications.
Moreover, it presents the quantitative information of the method usage percentages and application trend.

3) The advantages and limitations of AI algorithms are comprehensively investigated. Exemplary applications are provided for AI in each life-cycle stage, where the challenges and future research directions are discussed.

The rest of this paper is organized as follows. Section II presents the functions, methods, and milestones of AI in power electronics. The applications of AI in design, control, and maintenance are discussed in Section III, Section IV, and Section V, respectively. The outlook on the AI applications for power electronics is put forward in Section VI. Finally, conclusions are given in Section VII.

\section{Functions And Methods of ARtificial INTELLIGENCE FOR POWER ELECTRONIC SYSTEMS}

Fig. 2 gives a summary of the methods, functions, and applications of AI for power electronics. It can be seen that AI has been extensively applied to the three distinctive life-cycle phases of power electronic systems, including design, control, and maintenance.

As a functional layer between $\mathrm{AI}$ and power electronic applications, the essential functions of AI are categorized as optimization, classification, regression, and data structure exploration:

- Optimization: It refers to find an optimal solution maximizing or minimizing objective functions from a set of available alternatives given constraints, equalities, or inequalities that the solutions have to satisfy. For example, in the design task, optimization serves as a tool to explore an optimal set of parameters that maximize or minimize design goals with design constraints.

- Classification: It deals with assigning input information or data with a label indicating one of the $k$ discrete classes. Specifically, anomaly detection and fault diagnosis in maintenance is a typical classification task to determine fault labels with condition monitoring information.

- Regression: By identifying the relationship between input variables and target variables, the goal of regression is to predict the value of one or more continuous target variables given input variables. For example, an intelligent controller can be facilitated with a regression model between the input electrical signals and the output control variables.

- Data structure exploration: It consists of data clustering that discovers groups of similar data within a dataset, density estimation that determines the distribution of data within the input space, and data compression that projects high-dimensional data down to low-dimensional data for feature reduction. For example, in maintenance, the degradation state clustering is within the data structure exploration category.

According to the surveyed 444 relevant journal papers, Fig. 3 shows a Sankey diagram of application usage statistics of AI methods in the life-cycle of power electronic systems. Specifically, the percentages of AI application in the design, control, 


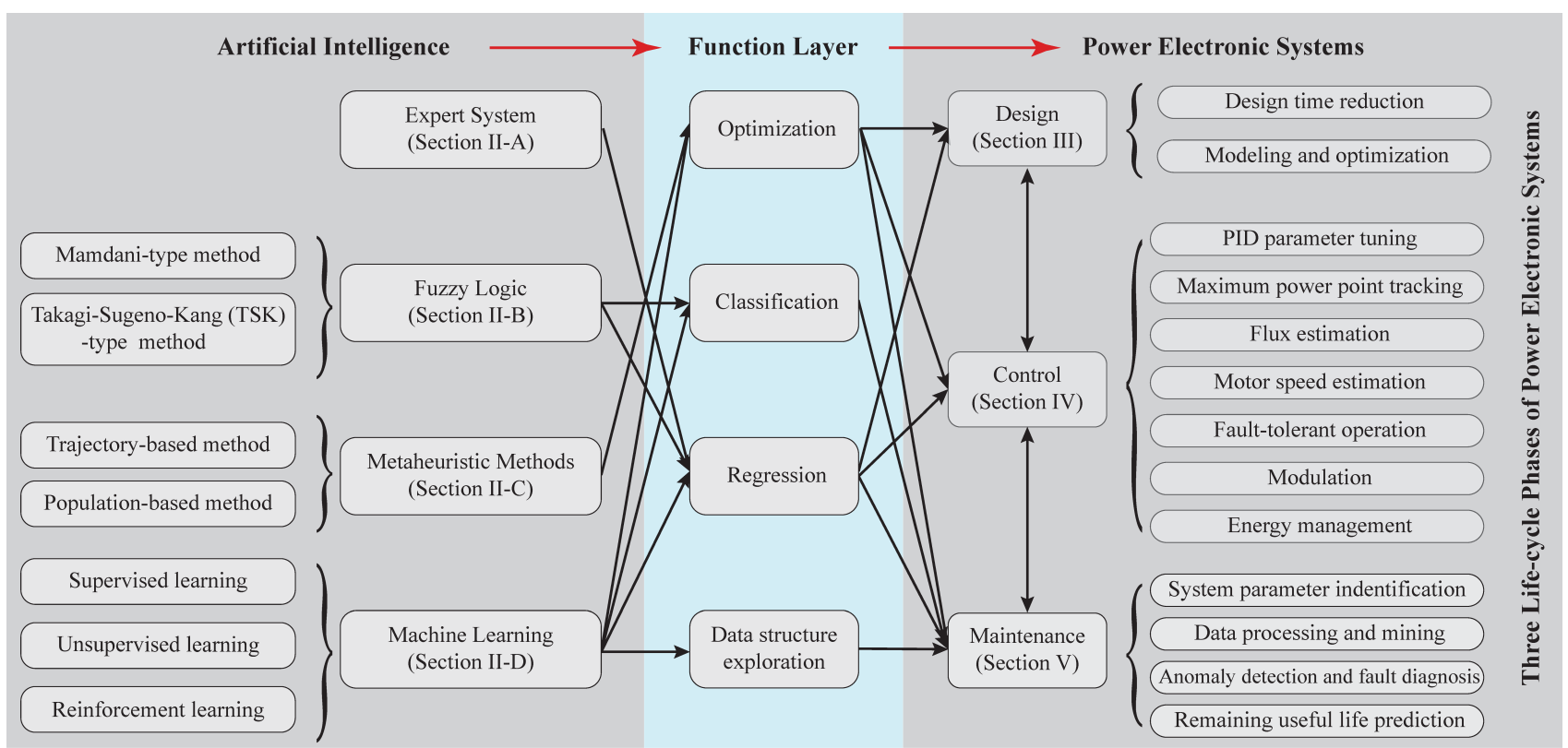

Fig. 2. The application of artificial intelligence in the life-cycle of power electronic systems. Section II-A implies that the relevant discussions are presented in part A of Section II.

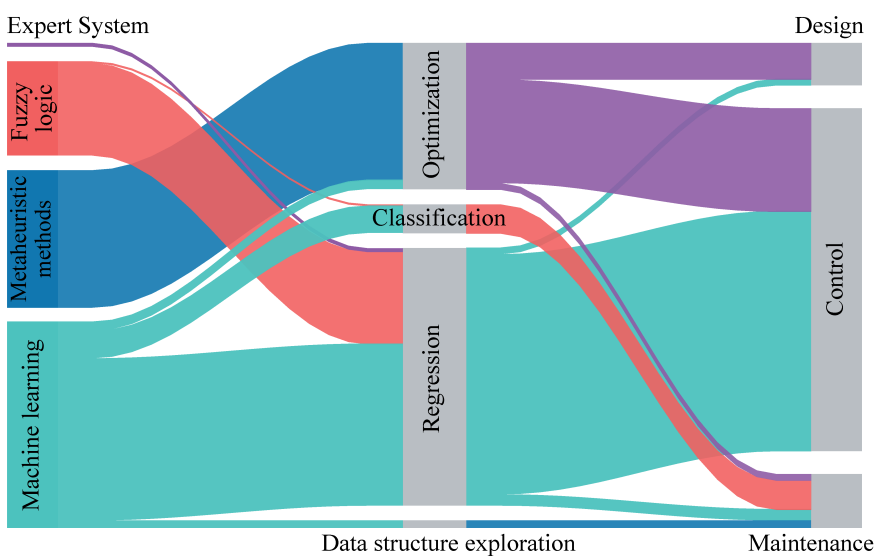

Fig. 3. Sankey diagram of AI methods and applications in each phase of the life-cycle of power electronic systems. The statistical usages and percentages are based on the data in Fig. 1.

and maintenance are $9.8 \%, 77.8 \%$, and $12.4 \%$, respectively. Regarding the functions, the percentages of optimization, classification, regression, and data structure exploration are $33.3 \%, 6.6 \%, 58.4 \%$, and $1.7 \%$. It shows that most of the tasks of $\mathrm{AI}$ in power electronics are essentially regression and optimization. The AI methods can be generally categorized as expert system, fuzzy logic, metaheuristic methods, and machine learning. Their application percentages are $0.9 \%$, $21.3 \%, 32.0 \%$, and $45.8 \%$, respectively. It suggests that the largest portion of $\mathrm{AI}$ in power electronics is with the machine learning. These methods will be detailed subsequently. Note that a comprehensive but still not exhaustive investigation is conducted. Only the relevant AI methods that are widely applied to power electronics are considered.

\section{A. Expert System}

Expert system is the earliest method in AI that is effectively implemented in industrial applications [17]. The expert system [24]-[27] is essentially a database that integrates the expert knowledge in a Boolean logic catalog, based on which the IF-THEN rules in human brain reasoning are simulated. It is an intelligent system simulating the inference process that answers the why-and-how inquires based on the database. The database is from either field expert experience or simulation data, facts, and statements. It can be continuously updated. The technical details of expert system are given in [17], and several exemplary applications can be found in [15], [28].

It is worth mentioning that the applications of expert system are as low as $0.9 \%$ according to the usage statistics in Fig. 3. It is because the expert system is generally based on system principles and rules, which relates strongly to the system of interest and lacks universality. It applies to well-defined domains only with solid expert rules. Besides, due to the rapid development of computational platforms, the functions of expert system can be replaced with other advanced AI methods (e.g., fuzzy logic and machine learning) with superior capabilities in inference and approximation.

\section{B. Fuzzy Logic}

Similar to expert system, fuzzy logic is also a rule-based method while it extends the Boolean logic into a multi-valued case. Fuzzy logic is an ideal tool to tackle system uncertainties and noisy measurements [29]-[31]. Instead of using the precise input crisp value directly, fuzzification is firstly performed with the fuzzy sets consisting of several membership functions to a range of 0 to 1 . The fuzzy input signals are then aggregated with fuzzy rules in the inference step. Defuzzification is subsequently performed on the inference result by considering the degree of fulfillment and output a crisp value. As a 
result, the crisp value is manipulated in a fuzzy space that completes nonlinear mapping between the input and output with elaborately designed principles.

In most applications, a fuzzy logic method mainly consists of four parts [30]: fuzzification, rule inference, knowledge base, and defuzzification. Firstly, fuzzification is performed on the input of linguistic variables with membership functions, including triangular, trapezoidal, Gaussian, bell-shaped, singleton, and other customized shapes. Secondly, the inference module integrates the signals together according to IF-THEN fuzzy rules in the knowledge base derived from expert experience. Thirdly, defuzzification is performed on the signal for output. One example of the fuzzy rule is

Antecedent: IF $X$ is Medium AND $Y$ is Zero,

Consequent: Then $Z$ is Positive.

For both the antecedent and consequent, the degree of fulfillment is determined by the membership functions. The type of fuzzy inference scheme is categorized as Mamdanitype [30], [32]-[35] and Takagi-Sugeno-Kang-type (TSKtype) [31], [36]-[38]. For the Mamdani-type fuzzy inference scheme, the membership function of the antecedent and the consequent are shape-based functions, e.g., triangular. For the TSK-type fuzzy inference scheme, the membership function of the antecedent part is identical to the Mamdani-type while that of the consequent is singleton at several constant values. Typically, more fuzzy sets are needed for the Mamdani-type scheme compared to the TSK-type scheme for the same task. Compared to the fuzzy terms in the Mamdani-type, the membership function in the TSK-type scheme can be functional type as either linear or constant, which is more powerful and accurate in nonlinear approximation. More theoretical details of fuzzy logic are discussed in [15], [39].

Note that expert experience plays a critical role in the design of the membership function and the fuzzy rule, and such a method is applicable to experts only in most cases. From this perspective, the prior information and expert experience can be coped with fuzzy logic and then incorporated with other AI techniques as a hybrid method.

\section{Metaheuristic Methods}

Once the optimization task of specific applications is formulated, the optimal solution can be obtained by either a deterministic programming method (e.g., linear or quadratic programming) or a non-deterministic programming method, i.e., metaheuristic method. The deterministic programming methods need to calculate the gradient and Hessian matrices [40], which is challenging for most of the optimization tasks in power electronics due to the complexity. Metaheuristic methods serve as a general end-to-end tool that needs less expert experience and is efficient and scalable for various optimization tasks.

The metaheuristic methods [12] are generally developed with inspirations of biological evolution, e.g., genetic algorithm [41] by process of natural selection, ant colony optimization algorithm (ACO) [42] by simulating ants in finding an efficient path for foods. The exploration of optimal solution is motivated by the trial-and-error process. The metaheuristic methods can be categorized as trajectory-based methods (tabu search method [43], simulated annealing method [44], etc.) and population-based methods (genetic algorithm, particle swarm optimization [45], ant colony optimization, differential evolution [46], immune algorithm [47], etc.). For the trajectorybased methods, each exploration stage includes only one candidate solution and it evolves into another solution according to a certain rule. The performance of this method is mainly based on the quality and efficiency of the rule. As a result, the convergence speed of the trajectory-based methods is generally slow and the final solution is prone to local rather than global solution for non-convex optimization tasks. For the populationbased methods, multiple candidate solutions are randomly generated. At each iterative exploration, these candidate solutions are diversified (e.g., crossover in the genetic algorithm) or incorporated and replaced with new candidate solutions to improve the quality of the population at the present generation. As a result, the suitability of the population is iteratively improved to approach the optimal solution. Compared to the trajectory-based methods, they are superior in the convergence speed, the global searching capability, and especially useful for large-scale optimization tasks. Nevertheless, the computational burden of the population-based methods is more intensive. This challenge needs to be considered for online application cases where efficiency and speed are of most significance. Table I shows a summary of the metaheuristic methods in the area of power electronics with their advantages and limitations. These metaheuristic methods are qualitatively compared in terms of several critical features including implementation simplicity, global convergence, convergence speed, and parallel capability.

Due to enormous advantages, most of the optimization tasks in power electronics are solved with the population-based methods. It can be seen from Table I that there are various population-based methods with the improved variants for optimization tasks in power electronics. They are developed and improved with different biological inspirations. In addition to the above widely applied metaheuristic methods, several other emerging approaches have been applied in a limited scale, e.g., biogeography-based optimization [72], crow search algorithm [73], grey wolf optimization [74], firefly optimization algorithm [16], bee algorithm [75], colonial competitive algorithm [76], teaching-learning-based optimization [77], etc. It is worth mentioning that the selection of the best method is not a simple task, which is application-dependent [12]. Genetic algorithm and particle swarm optimization are the two most popular metaheuristic methods applied to power electronics, as shown in Fig. 4. They are the fundamentals and representatives for evolutionary algorithms and swarm intelligence algorithms, respectively, based on which various variants are developed. Practitioners can choose the method considering its superiority according to Table I.

Note that there is no guarantee for a global optimum for metaheuristic methods, but the solution is generally satisfactory and acceptable for most practical applications. For more theoretical details of the metaheuristic methods, readers can refer to [16], [78]. 
Table I: The applications of metaheuristic methods in power electronics. Superior: +++ , intermediate: ++ , inferior: +

\begin{tabular}{|c|c|c|c|c|c|c|}
\hline \multirow[b]{2}{*}{ Type } & \multirow[b]{2}{*}{ Algorithms } & \multicolumn{4}{|c|}{ Advantages and Limitations } & \multirow[b]{2}{*}{ Exemplary Applications } \\
\hline & & $\begin{array}{l}\text { Implementation } \\
\text { Simplicity }\end{array}$ & $\begin{array}{l}\text { Global } \\
\text { Convergence }\end{array}$ & $\begin{array}{l}\text { Convergence } \\
\text { Speed }\end{array}$ & $\begin{array}{l}\text { Parallel } \\
\text { Capability }\end{array}$ & \\
\hline \multirow{5}{*}{$\begin{array}{l}\text { Population- } \\
\text { based } \\
\text { method }\end{array}$} & $\begin{array}{l}\text { Particle swarm } \\
\text { optimization (PSO) }\end{array}$ & + & +++ & + & Yes & $\begin{array}{l}\text { Design [45], [48], [49], Control } \\
\text { [50]-[56], Maintenance [57], } \\
\text { [58]. }\end{array}$ \\
\hline & $\begin{array}{l}\text { Genetic algorithm } \\
\text { (GA) }\end{array}$ & + & +++ & + & Yes & $\begin{array}{l}\text { Design [3], [41], [59]-[63], } \\
\text { Control [64]-[68], Maintenance } \\
\text { [69] }\end{array}$ \\
\hline & $\begin{array}{l}\text { Ant colony } \\
\text { optimization }(\mathrm{ACO})\end{array}$ & ++ & ++ & ++ & Yes & Design [70], Control [42] \\
\hline & $\begin{array}{l}\text { Differential } \\
\text { evolutionary (DE) }\end{array}$ & ++ & +++ & ++ & Yes & Control [46], [71] \\
\hline & $\begin{array}{l}\text { Immune algorithm } \\
\text { (IA) }\end{array}$ & ++ & ++ & ++ & Yes & Control [47] \\
\hline \multirow{2}{*}{$\begin{array}{l}\text { Trajectory- } \\
\text { based } \\
\text { method }\end{array}$} & Tabu search method & +++ & + & +++ & No & Design [43] \\
\hline & $\begin{array}{l}\text { Simulated } \\
\text { annealing method }\end{array}$ & +++ & ++ & +++ & No & Control [44] \\
\hline
\end{tabular}

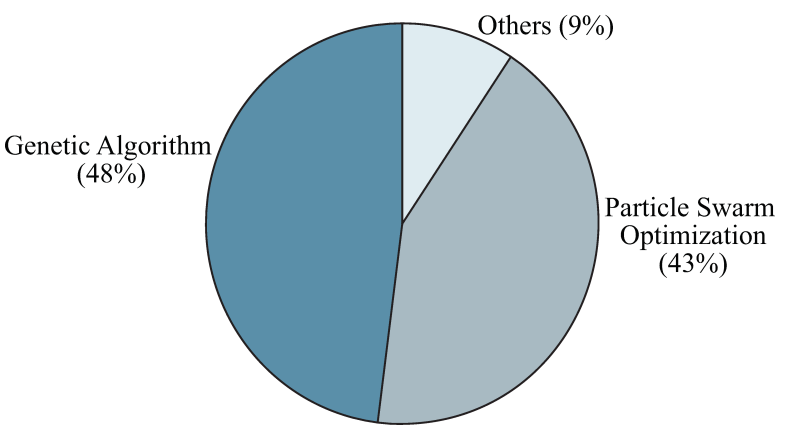

Fig. 4. Usage statistics of population-based metaheuristic methods in optimization of power electronics. The statistical results are obtained based on the data in Fig. 1.

\section{Machine Learning}

Machine learning is designed to automatically discover principles and regularities with experience from either collected data or interactions by trial-and-error. For applications in power electronics, it is categorized as supervised learning, unsupervised learning, and reinforcement learning.

1) Supervised Learning: With the training dataset consisting of input-and-output pairs, the supervised learning aims to establish the mapping and functional relationships between the inputs and outputs implicitly. This feature is especially useful for cases in power electronics where system models are challenging to formulate. Generally, the tasks of the supervised learning include classification and regression. For classification, its output of the input-and-output pairs in the training dataset deals with a finite number of discrete categories to be labeled. For example, the fault diagnosis for a multilevel inverter [94] is a typical classification task where the discrete fault label needs to be identified given the input fault information. For a regression task, the output of the input-andoutput pairs consists of one or more continuous variables. An example of regression is the remaining useful life prediction of IGBTs [114] where the output, i.e., the residual useful lifetime, is a continuous variable. Once the model is trained, it is ready to evaluate new data points that differ from the training dataset. The model capability in dealing with new data points, i.e., the ones in the testing dataset, is termed as the generalization. Since the training dataset comprises only a limited amount of possible input-and-output pairs in most cases, its generalization on new inputs is one of the most critical performance factors of supervised learning methods.

Generally, supervised learning methods can be categorized into connectionism-based methods (i.e., neural network method), probabilistic graphical methods, and memory-based methods (i.e., kernel method). For neural network methods, knowledge learned from the training dataset is facilitated and transferred as the connection weights and structures of the network. Numerous research has been devoted to improving the performance of neural network methods. These improvements are from two aspects for applications in power electronics. The first aspect deals with enabling the uncertainty capability in handling the noisy signal of the neural network to improve the method robustness. This feature is facilitated by integrating the fuzzy logic into the neural network as the fuzzy neural network or its variants (e.g., adaptive neurofuzzy inference system (ANFIS) [101]). The second aspect is for dynamic-performance improvement of the neural network to tackle time-series dataset cases, e.g., intelligent controller, remaining useful life prediction. Compared to the conventional neural network where the network weights are independent, the transient performance is facilitated by sharing weights between different layers and network cells. The weight sharing can be implemented either in a shallow scale with a convolutional structure (e.g., 1-D convolutional neural network, Timedelayed neural network (TDNN) [114]), or in full and deep scale by using a recurrent unit as recurrent neural network [105]. Generally, the modeling capability of recurrent unit implementation is superior to the one with a convolutional structure. More theoretical details of the neural network methods are discussed in Chapter 5 of [1] and [13], [14].

The probabilistic graphical methods obtain knowledge from the data by using a diagrammatic representation of inputand-output pairs. The diagrammatic representation implies the conditional dependence relationship between the decision variables. The underlying relationship in the model is formulated in the Bayesian framework [1] and can be inferred in 
Table II: Supervised learning methods and the applications to power electronics.

\begin{tabular}{|c|c|c|c|c|}
\hline Type & Method & Variants & Advantages and Limitations & Exemplary Applications \\
\hline \multirow{9}{*}{ 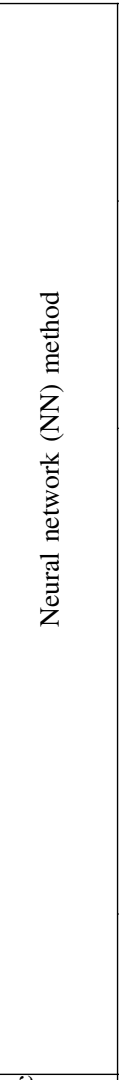 } & \multirow[t]{2}{*}{$\begin{array}{c}\text { Conventional } \\
\text { NN }\end{array}$} & $\begin{array}{l}\text { Feed-forward neural } \\
\text { network (FFNN) }\end{array}$ & N/A (Not applicable) & $\begin{array}{l}\text { Design [79], [80], Control [4], } \\
\text { [51], [81]-[85], Maintenance } \\
{[86]-[97]}\end{array}$ \\
\hline & & $\begin{array}{l}\text { Radial basis function } \\
\text { network (RBFN) }\end{array}$ & $\begin{array}{l}\text { Compared to FFNN: } \\
\text { - Simple network structure } \\
\text { - Higher speed of training }\end{array}$ & Control [50], Maintenance [98] \\
\hline & \multirow[t]{2}{*}{$\begin{array}{l}\text { NN with } \\
\text { fuzzy logic }\end{array}$} & $\begin{array}{l}\text { Fuzzy neural network } \\
\text { (FNN) }\end{array}$ & $\begin{array}{l}\text { Compared to conventional NN: } \\
\text { - Capability of handling uncertainty } \\
\text { - Incorporation of expert experience } \\
\text { - Higher speed of training }\end{array}$ & Control [46], [71], [99], [100] \\
\hline & & $\begin{array}{l}\text { Adaptive neuro-fuzzy } \\
\text { inference system } \\
\text { (ANFIS) }\end{array}$ & $\begin{array}{l}\text { Compared to FNN: } \\
\text { - Automatic fuzzy-rule generation with less expert } \\
\text { experience }\end{array}$ & $\begin{array}{l}\text { Control [101], Maintenance } \\
{[102],[103]}\end{array}$ \\
\hline & \multirow{4}{*}{$\begin{array}{l}\mathrm{NN} \text { with } \\
\text { recurrent unit }\end{array}$} & $\begin{array}{l}\text { Recurrent neural } \\
\text { network (RNN) or } \\
\text { Elman NN (ENN) }\end{array}$ & $\begin{array}{l}\text { Compared to conventional NN: } \\
\text { - Better transient and dynamic capability } \\
\text { - Better sensitivity } \\
\text { - Slow speed in training }\end{array}$ & $\begin{array}{l}\text { Control [52], [53], [71], [99], } \\
\text { [104]-[107], Maintenance [108] }\end{array}$ \\
\hline & & $\begin{array}{l}\text { Nonlinear } \\
\text { autoregressive } \\
\text { network with } \\
\text { exogenous inputs } \\
\text { (NARX) } \\
\end{array}$ & $\begin{array}{l}\text { Compared to RNN: } \\
\text { - Higher speed of training } \\
\text { - Better generalization capability } \\
\text { - Better capability in dealing with long-term } \\
\text { dependence }\end{array}$ & $\begin{array}{l}\text { Design [109], Maintenance } \\
\text { [110] }\end{array}$ \\
\hline & & $\begin{array}{l}\text { Echo state network } \\
\text { (ESN) }\end{array}$ & $\begin{array}{l}\text { Compared to RNN: } \\
\text { - Only hidden-to-output weights need to determine } \\
\text { - less severe issue of gradient explode and vanish }\end{array}$ & Maintenance [111], [112] \\
\hline & & $\begin{array}{l}\text { Long short-term } \\
\text { memory (LSTM) }\end{array}$ & $\begin{array}{l}\text { Compared to RNN: } \\
\text { - Most effective structure for practical application } \\
\text { - No issue of gradient exploding and vanishing }\end{array}$ & Maintenance [113] \\
\hline & $\begin{array}{l}\mathrm{NN} \text { with } \\
\text { convolutional } \\
\text { structure }\end{array}$ & $\begin{array}{c}\text { Time-delayed neural } \\
\text { network (TDNN) } \\
\text { (also termed as 1-D } \\
\text { convolutional NN } \\
(\mathrm{CNN}))\end{array}$ & $\begin{array}{l}\text { Compared to conventional NN: } \\
\text { - Partial weight sharing for transient performance } \\
\text { improvement for specific applications } \\
\text { Compared to RNN: } \\
\text { - Capability of time-series modeling is weaker }\end{array}$ & $\begin{array}{l}\text { Control [14], Maintenance } \\
{[114],[115]}\end{array}$ \\
\hline 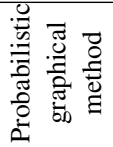 & N/A & Bayesian Networks & $\begin{array}{l}\text { Compared to NN methods: } \\
\text { - Better interpretability } \\
\text { - Computationally intensive } \\
\text { - Probabilistic output with uncertainty quantification }\end{array}$ & Maintenance [96], [116]-[118] \\
\hline \multirow{3}{*}{ 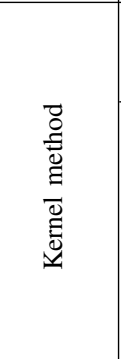 } & $\begin{array}{l}\text { Conventional } \\
\text { kernel method }\end{array}$ & Gaussian processes & $\begin{array}{l}\text { Compared to NN methods: } \\
\text { - Probabilistic output with an uncertainty } \\
\text { quantification }\end{array}$ & Maintenance [119], [120] \\
\hline & \multirow[b]{2}{*}{$\begin{array}{l}\text { Sparse kernel } \\
\text { method }\end{array}$} & $\begin{array}{l}\text { Support vector } \\
\text { machine (SVM) }\end{array}$ & $\begin{array}{l}\text { Compared to conventional kernel method: } \\
\text { - Better approximation capability with small dataset } \\
\text { - Better computational efficiency }\end{array}$ & $\begin{array}{l}\text { Control [121], [122], } \\
\text { Maintenance [7], [74], [96], } \\
{[118],[123]-[126]}\end{array}$ \\
\hline & & $\begin{array}{l}\text { Relevance vector } \\
\text { machine (RVM) }\end{array}$ & $\begin{array}{l}\text { Compared to SVM: } \\
\text { - Much sparser than SVM while maintaining } \\
\text { comparable generalization capability } \\
\text { - Probabilistic output with uncertainty quantification } \\
\text { - Training time is generally longer than SVM }\end{array}$ & Maintenance [127], [128] \\
\hline
\end{tabular}

a probabilistic way. Thus, the interpretability of the model is much better compared to neural network methods. Besides, the probabilistic graphical model is superior in dealing with uncertainty and incomplete knowledge. One of the typical probabilistic graphical methods is the Bayesian network [117]. More theoretical details of the probabilistic graphical methods are given in Chapter 8 of [1].

For the neural network methods and the graphical methods, the training dataset is discarded when the training is completed. While the training dataset in kernel methods is kept and used in the testing stage, and the learned knowledge is facilitated as the identification of critical data points (e.g., support vectors in support vector machine [126]) or subset in the training dataset. One typical kernel method is Gaussian processes, which has been applied to the remaining useful life prediction of IGBTs in [119]. Note that the conventional kernel methods (e.g., Gaussian processes) are computationally intensive due to the whole training dataset is applied to the testing stage. To avoid the excessive computational burden, sparse solutions are proposed as support vector machine (SVM) and relevance vector machine (RVM), where the parameter estimation is improved based on Bayesian methods. With the sparse solution, only a subset of the training dataset is applied to the testing stage and thus it is more efficient compared to the conventional kernel methods. More theoretical details of the kernel methods are discussed in Chapters 6 and 7 of [1]. Generally, the requirement of the training dataset for the kernel methods is lower than the neural network methods. Therefore, the kernel methods are more suitable for the cases with a small dataset. While due to the training dataset is needed in the testing stage, the memory requirement of the kernel methods 
Table III: Unsupervised learning methods and the applications to power electronics.

\begin{tabular}{|c|c|l|l|}
\hline Function & Method & Advantages and Limitations & Exemplary Applications \\
\hline \hline \multirow{2}{*}{ Clustering } & $k$-means & $\begin{array}{l}\text { - Simple implementation } \\
- \text { Sensitive to outliers }\end{array}$ & $\begin{array}{l}\text { Control [129], Maintenance } \\
{[118],[130]-[132]}\end{array}$ \\
\cline { 2 - 4 } & $\begin{array}{l}\text { Self-organizing } \\
\text { maps (SOMs) } \\
\text { Data compression }\end{array}$ & $\begin{array}{l}\text { Maintenance [133], Control } \\
- \text { Less sensitive to initial parameter selection } \\
\text { - Less sensitive to outliers }\end{array}$ \\
\hline $\begin{array}{c}\text { Principal } \\
\text { compont analysis } \\
\text { (PCA) }\end{array}$ & $\begin{array}{l}- \text { Flexible framework with various improvements, } \\
\text { e.g., kernel PCA, Bayesian PCA, etc }\end{array}$ & $\begin{array}{l}\text { Control [134], Maintenance } \\
{[117],[127],[132],[135],[136]}\end{array}$ \\
\hline
\end{tabular}

is higher than the neural network methods. The involvement of the training dataset also limits the speed performance at the testing stage. It should be considered for online applications where the execution time is critical, e.g., control application.

As a result, Table II shows a summary of the supervised learning methods and their variants in power electronics, in terms of the advantages, limitations, and exemplary applications.

2) Unsupervised Learning: Compared to the supervised learning where the dataset is input-and-output pairs, unsupervised learning has no output data for the learning target during the learning process. Generally, the tasks of unsupervised learning in applications of power electronics can be categorized as data clustering and data compression.

For the data clustering, it explores the regularities from the smeared dataset and partitions the dataset into several different groups or clusters according to their similarities. In this way, the data characteristics within the same cluster are similar to each other and different from the ones in other clusters. One typical data clustering application is the identification of the discrete health state from the continuous degradation data [131] in the condition monitoring of power electronic converters. The purpose of the data compression is to eliminate excessive information in the dataset to reduce the number of features of the dataset. For example, using principal component analysis (PCA) [127], a reduced representation of the dataset is obtained with a much fewer number of features, which yet maintain the integrity of the dataset.

Generally, these unsupervised learning algorithms serve as the data-preprocessing before it goes to the subsequent analytics (e.g., fault diagnosis). Although this step is optional, it is beneficial to reducing the computational burden and improving the analytics accuracy. Table III gives a summary of typical unsupervised learning methods for power electronic applications. More unsupervised learning methods and theoretical details can be found in [137].

3) Reinforcement Learning : In contrast to the supervised learning and the unsupervised learning, reinforcement learning (RL) does not require a training dataset. Instead, it aims to find a suitable action strategy that maximizing the reward for a specific task, which is essentially a dynamic programming or optimization task. This goal-oriented strategy is formulated from interactions with systems or simulation models by a trialand-error process [138]. In this way, it accumulates experience progressively and learns a specific strategy that maximizes the predefined goal. Theoretically, RL is a Markov decision process [139]. The training of RL aims to develop a Q-table

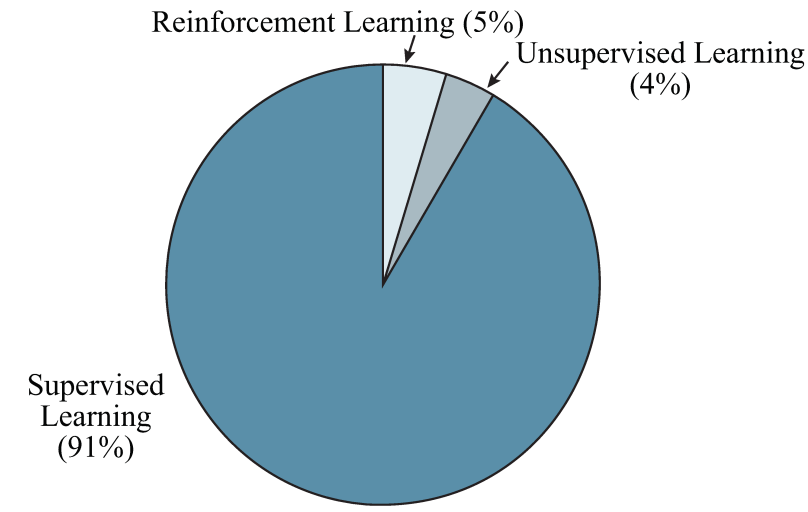

Fig. 5. Usage statistics of machine learning methods in power electronic systems. The statistical results are obtained based on the data in Fig. 1 .

in terms of an action selection policy, which can maximize the total expected rewards over the future. The Q-table is an informative policy matrix that records the optimal action to be taken given the particular condition variables. More theoretical details of RL can be found in [139]. One application example is the MPPT [5], [6], [140]. Note that RL obtains the experience from the interactions between systems instead of existing datasets. It is thus more favorable for the cases where the system is with less knowledge or its model is challenging to formulate.

As a summary, Fig. 5 presents the usage statistics of the machine learning methods. Supervised learning is dominantly applied to power electronics. The reason is that the supervised learning is a versatile tool, which is typically the central part of the majority of machine learning-related applications in power electronic systems.

\section{E. Timeline of Relevant AI methods and Applications in Power Electronics}

Fig. 6 summarizes the milestones of the relevant AI methods and their applications in power electronics. It includes the year when the algorithm is first proposed, the first application in power electronics, the milestones of relevant AI algorithms, and applications in terms of each method. It should be noted that the information is to the best knowledge of the authors. Also, the timeline is not extensive to include all of the existing AI algorithms. Instead, only the ones that show great potentials in power electronics are included. According to Fig. 6, it can be noted that:

1) The application of both expert system and fuzzy logic is moderate nowadays, especially for the expert system. 


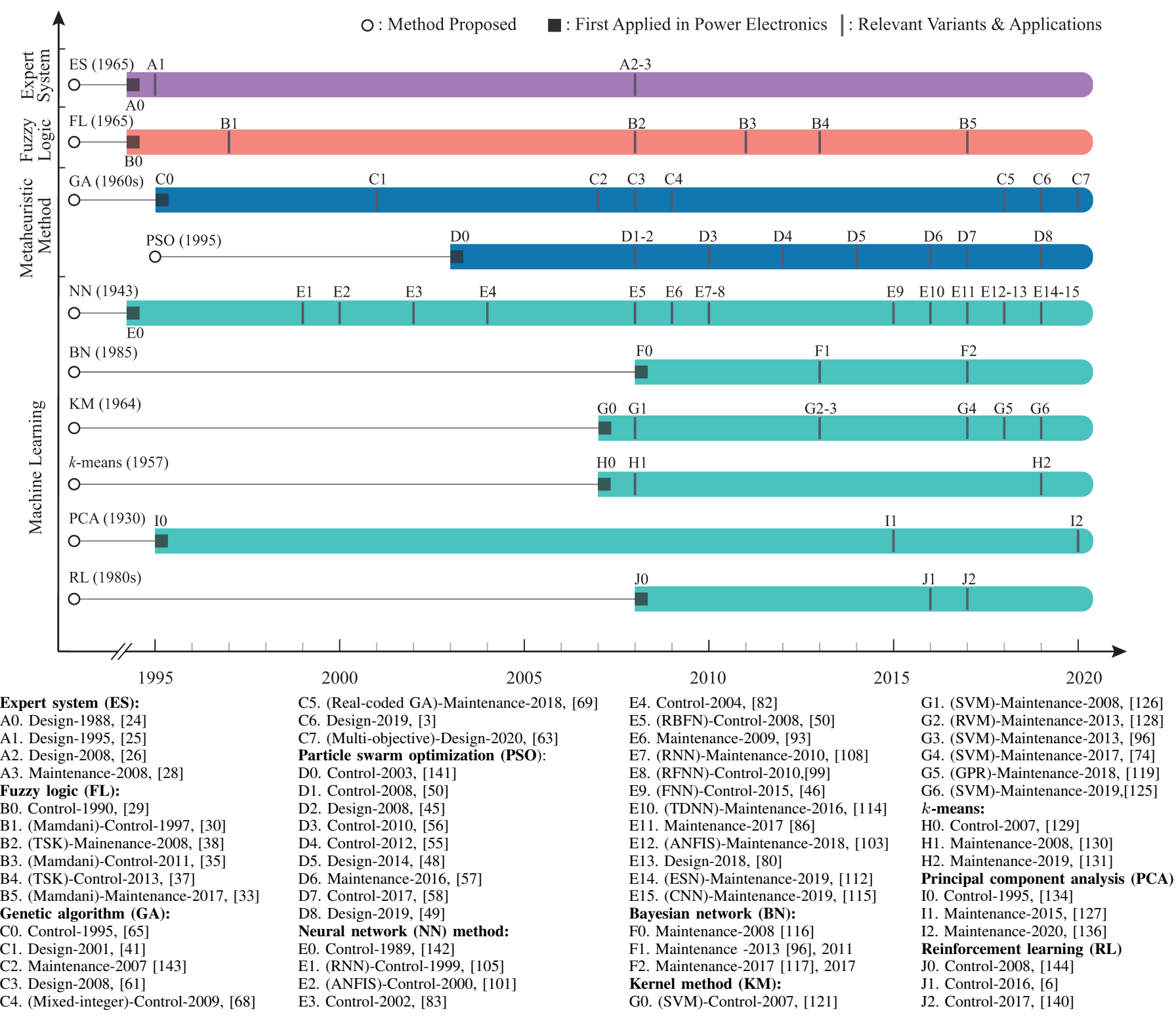

Fig. 6. Timeline of relevant AI methods and applications in power electronics. The milestones are identified considering the significant algorithm variants and the relevant applications. It is organized as the form of (significant variants)-application-year. Significant variant is specifically indicated. Otherwise, it is a standard algorithm.

Before the 2000s, their practical implementations are developed in the presence of the limited performance of computing hardware, which has been significantly improved to date. This rapid development of computing hardware facilitates and accelerates the implementation of other more powerful AI methods for replacing expert system and fuzzy logic.

2) Metaheuristic methods are continuously evolving and applied to power electronics. They are used for a complete task or a key step jointly with other machine learning methods.

3) Neural network methods are the most active area for AI applications for power electronics. The reason is twofold. Firstly, the significant development of computing hardware unleashes the potentials of neural network methods in dealing with complex tasks in power elec- tronic systems. Secondly, the structure of neural network is quite flexible to incorporate other AI methods for performance improvement, implying numerous method variants.

4) There is an increasing trend of applications with kernel methods and probabilistic graphical models. It is because most of these methods are formulated within the Bayesian framework, which possesses better generalization and interpretability. Moreover, their computational burden can be well tackled with the platforms to date.

5) RL is the latest frontier of the machine learning methods applied to power electronics, facilitated by the rapid development of computing hardware.

It can be noted from Figs. 2, 3, and 6 about the comparisons for different AI methods:

1) Both metaheuristic methods and machine learning can 
be applied to optimization tasks. Specifically, machine learning-based optimization (i.e., reinforcement learning) focuses on the dynamic optimization involved with the decision-making (e.g., MPPT). Metaheuristic method is generally applied to the static optimization (e.g., heatsink design).

2) Both fuzzy logic and machine learning can be exploited for classification tasks. Generally, machine learning is more accurate and flexible than fuzzy logic.

3) The regression task can be implemented with expert system, fuzzy logic, and machine learning. The implementation of expert system is simple but less powerful compared to fuzzy logic and machine learning. The implementation of fuzzy logic needs expert experience. Machine learning is the most popular method and various algorithm variants have been developed. It can be incorporated with fuzzy logic for performance improvement.

4) Only machine learning can be applied to the task of data structure exploration.

The following three sections discuss the applications of the above introduced AI methods in the design, control, and maintenance phases of power electronic systems, respectively.

\section{DESIGN}

Design in power electronics encompassing topology selection, component sizing, circuit synthesis, reliability considerations, etc., is essentially an optimization task [145]. A typical procedure for the design of power electronic systems comprises four steps:

1) Objective formulation: Objective functions are desirable design goals to be maximized or minimized. Generally, the design goals in power electronics include component parameter [41], weight [146], volume [147], cost [146], heatsink pattern [3], area [148], power loss [62], etc. It is crucial for formulating the required or desired design requirements to several explicit mathematical expressions as a single objective as given in (1) or multiple objectives as given in (2) [12], [145]:

$$
\begin{array}{ll} 
& \max _{\mathbf{x}} f(\mathbf{x}), \\
& \max _{\mathbf{x}} \mathbf{w}^{T} \mathbf{f}(\mathbf{x}), \max _{\mathbf{x}} \mathbf{f}(\mathbf{x}), \\
\text { s.t. } & g(\mathbf{x}) \leq 0, h(\mathbf{x})=0, \mathbf{x} \in\left[\mathbf{x}_{l}, \mathbf{x}_{u}\right] .
\end{array}
$$

where $g(\mathbf{x})$ and $h(\mathbf{x})$ are inequalities and equalities, respectively. $\mathbf{x}_{l}, \mathbf{x}_{u}$ are the lower and the upper boundaries for decision variables $\mathbf{x}$, respectively. Here the maximization is the goal, which can simply be applied to the minimization case. Note that for multiple objectives in (2), it can be either solved by maximizing a scalar function $\mathbf{w}^{T} \mathbf{f}(\mathbf{x})$ by weighting multiple objectives together or by optimizing objective vector $\mathbf{f}(\mathbf{x})$ directly, where Pareto front [62] can be applied to determine the optimal solution, e.g., the non-dominated sorting genetic algorithm method for multiobjective design optimization of power modules in [60].
2) Constraint space: The constraint space defines feasible space, boundary, relationship, and limitation that the objective function is subjected to. These constraints include either linear or nonlinear equalities and inequalities. They are derived from the practical design requirements, e.g., geometry, volume, lifetime characteristics, cost, etc.

3) Solution exploration: The defined optimization problem is to maximize (or minimize) objective functions by adjusting the decision variables in the constraint spaces. AI methods, especially the metaheuristic methods, can be applied to this step.

4) Performance evaluation: The candidate solution can be tested against the predefined objectives by using simulation, hardwire-in-the-loop testing, prototype experiment, etc. The results can be returned to previous steps for further performance improvement and optimization.

Instead of a sequential procedure, the design task is an iterative trial-and-error process. Based on the evaluation at each step, the task may be reformulated, e.g., adjusting the objectives, modifying the constraint space, reconfiguring the programming methods, etc. For conventional design in power electronics, it is time-consuming and needs multiple iterative steps. For example, the component alignment and the model selection rely on expert experience and intuition without ample quantitative reference. In this way, the design performance will converge slowly to the required standards. This drawback can be mitigated by AI methods. They can be applied to Step 1) objective formulation for the design time reduction, and Step 3) solution exploration for the modeling and optimization.

\section{A. Design Time Reduction}

The formulation of design objective needs to be improved if its evaluation is computationally intensive. One application of AI methods is a surrogate model in the objective formulation to reduce the computational effort. The surrogate model yields an identical behavior to the system dynamics that are challenging to formulate or need intensive computational efforts to characterize. In the iterative design process, AI-based surrogate model serves as a replacement that significantly reduces the computational effort.

As an application of Design for Reliability (DfR), in [80], two feed-forward neural networks are applied to the automated reliability design of power electronic systems. The first feedforward neural network serves as a surrogate model emulating thermal characteristics of power converters, by which the design parameters can be mapped to the information of junction temperature variations. Subsequently, the second feed-forward neural network is applied to map the annual mission profiles (e.g., annual solar irradiation and ambient temperature) to the annual lifetime consumption. In this way, the nonlinear relationship between the designed parameters and the annual lifetime consumption is quantitatively characterized, which can accelerate the iterative design process.

Another example of AI for DfR of power electronic systems is given in [109]. With superior capability in tackling timeseries data, a nonlinear autoregressive network with exogenous inputs (NARX) is applied to the thermal modeling of power 


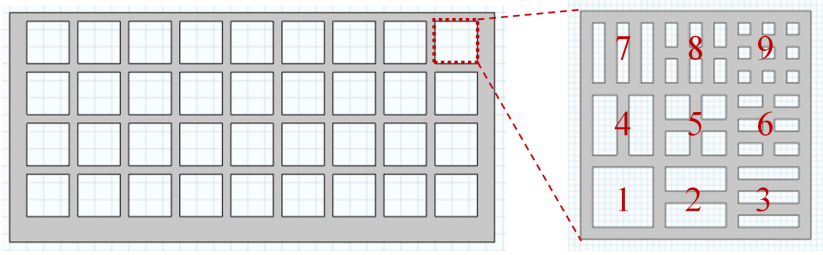

Fig. 7. Nine different cell patterns for each blank cell [3]. A genetic algorithm is applied to determine the optimal combination of different cell patterns for the heatsink design for minimizing the junction temperature.

electronic systems considering the thermal cross-coupling effects. The proposed NARX-based thermal model can be completed within around $109 \mathrm{~s}$, which is a significant efficiency improvement compared to the $1005 \mathrm{~s}$ of the conventional model. The error between the temperature estimated by the NARX-based thermal model and the actual measurement is less than $1{ }^{\circ} \mathrm{C}$. Experimental results indicate that the NARXbased thermal model can replace the conventional model with less testing efforts and much less computational burden.

In [79], considering the electrothermal interactions, a feedforward neural network is applied to construct the component behavior model of MOSFETs without any in-depth knowledge of the device structure. Under the static state, the complicated nonlinear and temperature-dependent characteristics between the variables, including drain-to-source voltage $V_{\mathrm{DS}}$, gate-tosource voltage $V_{\mathrm{GS}}$, junction temperature $T_{j}$, and the output current $I_{\mathrm{D}}$ are established by using the neural network. This compact model can drastically accelerate the design simulation process with a comparable accuracy.

\section{B. Modeling and Optimization}

The modeling and optimization of power electronic systems is about specifying circuit topology, component model, component parameter, etc, such that system dimension, weight, operating frequency, etc., will result as optimal characteristics (e.g., power loss, power density) given design constraints [12]. Specifically, the optimization method is applied to the solution exploration to provide an overall optimal configuration, where metaheuristic methods in AI can be exploited. As mentioned, the selection of a suitable metaheuristic method depends on the specific application. Several exemplary applications are given as follows.

In [3], genetic algorithm (GA) is combined with finite element analysis for the automated heatsink design of a 50 $\mathrm{kW}$ three-phase inverter. As shown in Fig. 7, GA is applied to optimize the combination of nine customized patterns to formulate a complex cell pattern of heatsink. The goal is to minimize the junction temperature of power semiconductor devices. Compared to the conventional design with a regular cell pattern, the proposed method formulates a heatsink solution with $27 \%$ less in size and $6 \%$ lower in junction temperature.

In [62], the design of a $500 \mathrm{~kW}$ solar power-based microgrid system is formulated as a multiobjective optimization task, which maximizes the average power distribution and minimizes the system weight simultaneously. It explores the optimal values of four microgrid parameters, including battery voltage, PV maximum power, PV maximum power point voltage, and number of panels per string. The GA combining with the Pareto front is applied to solve the multiobjective optimization task. Besides, there is a specifically improved variant of $\mathrm{GA}$ for the multiobjective optimization task, i.e., non-dominated sorting genetic algorithm II (NSGA-II) [63].

In [45], the particle swarm optimization (PSO) is applied to the circuit synthesis of a power electronic circuit, where the optimal values of components are explored to fulfill the design goals of better static and dynamic performance. For this specific case, the simulation indicates that the PSO yields a superior solution with less computational effort compared to GA.

In [70], the ant colony optimization (ACO) is applied to determine the optimal component values in a power electronic circuit, where the conventional ACO is extended to facilitate the optimization with continuous component values and accelerate the optimization process. Moreover, the component tolerance is incorporated into the optimization, which makes the proposed method more beneficial to practical applications.

\section{CONTROL}

Essentially, control applications with AI methods in power electronic systems can be categorized as the optimization and the regression. Similar to the optimization in the design phase, the optimization-related tasks in control applications are also dealing with metaheuristic methods. Several representative applications are given below.

In [64], a GA is applied to the PID tuning of a programming logic controller, where the optimization goal is to minimize the error between the ideal step and ramp responses and the ones initialized with proportional term $K_{p}$, integral term $K_{I}$, and derivative term $K_{D}$ found by GA. Experimental analysis indicates that the output performance of the optimized controller is very close to the ideal step and ramp responses.

In [42], to overcome the challenges of multiple maximum power points in partially shaded situations for PV systems, an ACO-based MPPT method is proposed. It is compared with conventional methods, including constant voltage tracking, perturb \& observe, and PSO. The experimental results indicate that the ACO-based MPPT method is superior in global convergence and robustness to various shading patterns.

In [47], in a single-phase full bridge inverter, an IA is applied to find the optimal sinusoidal pulse-width modulation (PWM) control sequences of four switches minimizing the total harmonic distortion (THD) of the output waveforms. The experiment indicates that the THD by using IA is $0.79 \%$, which is superior to that of the conventional control method of hysteresis current PWM with $1.23 \%$ and the GA solution with $0.99 \%$. Moreover, the IA is superior to the GA in convergence speed. More examples of optimization-related control applications can be found in [12].

The regression-related tasks in control applications are dealing with the nonlinear mapping of system inputs and outputs in a static or dynamic way. Specifically, it is concerned with regulating systems to ensure intended performance output with system principles. Several limitations of conventional methods are identified: 


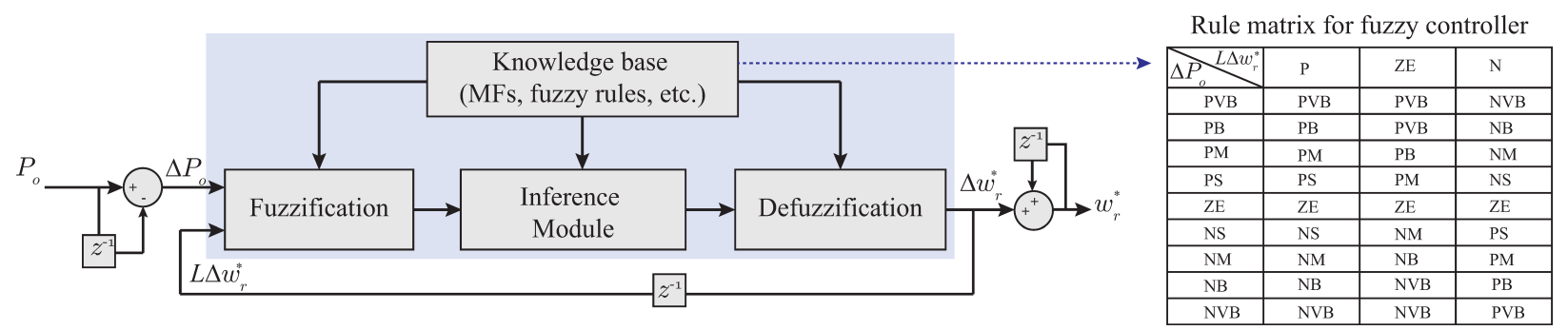

Fig. 8. Fuzzy logic-based controller for a variable-speed wind generation system [30]. MFs: Membership functions. In the rule matrix table, P: positive, V: very, B: big, M: medium, ZE: zero, N: negative.

1) The controller configuration requires in-depth knowledge of system control principles, which are challenging and even infeasible for complex cases. It is timeconsuming for complex systems to consider the timevarying and piecewise-linear characteristics, where the controller is generally optimized at several critical operational points rather than the full operational area, resulting in a sub-optimal solution.

2) Once the controller is installed, it operates in a static way with limited adaptability, suggesting that it is only applicable to time-invariant systems. Nevertheless, when environmental and operational conditions change, the controller will be less robust to system parameter shifts and the control performance is likely to deteriorate.

3) From the efficient control perspective, an ideal controller must be able to cope with parameter tolerances with a fast transient response to maintain system stability. However, such a desired feature cannot be well fulfilled.

These limitations can be mitigated with AI methods. For the regression-related task in control applications, it is organized in terms of fuzzy logic, neural network, and reinforcement learning, respectively.

\section{A. Fuzzy Logic-based Controller}

Fuzzy logic-based methods have been widely applied to the control of power electronic systems, e.g., speed control [30], MPPT [35], energy management [149], to name a few.

In [30], a control strategy with three fuzzy logic controllers is developed for a variable speed wind generation system. The structure of the generator speed programming controller is given in Fig. 8. The control variables include the increment of the output power $\Delta P_{o}$ and the last variation of speed $L \Delta w_{r}^{*}$. The controller outputs the variation of speed $\Delta w_{r}^{*}$ to adjust the generator speed for a maximum wind power output. The Mamdani-type fuzzy logic is applied and the information is aggregated according to the rule matrix table, e.g., "IF $\Delta P_{o}$ is PS AND $L \Delta w_{r}^{*}$ is ZE, THEN $\Delta w_{r}^{*}$ is PM". The membership functions are iteratively tuned by the system simulation and experiment. Similar Mamdani-type fuzzy logic controller for the primary frequency regulation of a wind farm can be found in [34].

In [36], a fuzzy logic controller is proposed for regulating the speed of a switched reluctance motor based on TSK fuzzy logic by approximating an ideal control law. The parameter is tuned by using the Lyapunov stability theorem to ensure system stability. The experimental analysis demonstrates that the developed adaptive TSK-type controller outperforms the conventional fuzzy logic controllers and the PI controller. A similar TSK-type controller can be found in [31] for approximating the typical sliding mode control curve for integrated LED drivers. It is computationally efficient and implemented on a low-cost platform.

Although the fuzzy logic controller can handle the system uncertainty, similar to conventional methods such as PID, there is no internal updating mechanism and thus the adaptability is limited [50]. Also, it can be seen that the design of membership functions and fuzzy rules require expert experience, which highly limits the method practicality. Thus, such a method is applicable to experts only in most cases. Nevertheless, from this perspective, the expert experience can be coped with fuzzy logic and then incorporated with other AI techniques as a hybrid method, as discussed later.

\section{B. Neural Network-based Controller}

As a black-box technique, neural network can approximate a wide range of nonlinear functions to arbitrary accuracy. With few requirements on system knowledge, the NN-based controller possesses several advantages such as robustness, model-free, dynamic, adaptive, universal approximation, etc.

1) Conventional Neural Network: The most widely used neural network in power electronics is the feed-forward neural network (FFNN) (or backpropagation neural network) with a feed-forward multilayer and a backpropagation topology [14]. The respective applications essentially exploit the property of static nonlinear mapping of the FFNN.

In [82], an FFNN is applied to the waveform processing and delayless filtering. With two cases of variable frequency and variable magnitude, it indicates that the FFNN can convert $m$ phase waveform with an arbitrary shape into the $n$-phase waveform with various characteristics of magnitude and frequency. The FFNN-based waveform processing method provides a simplification of the hardware implementation. Moreover, additional single processing functions can be embedded easily due to the structure flexibility.

In [83], the space vector PWM (SVPWM) for a threelevel voltage-fed inverter is implemented with an FFNN. The input of the neural network is the sampled command phase voltages and the output is the pulse width patterns of SVPWM. The training data are generated by the simulation with an SVPWM algorithm. By comparing with a conventional DSPbased SVPWM solution, the performance of the FFNN-based 


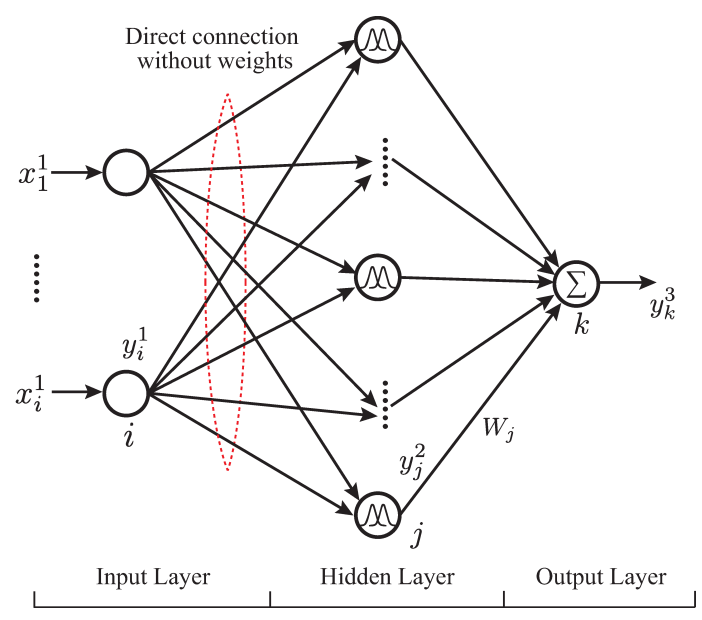

Fig. 9. Structure of a radial basis function network (RBFN) with three layers [50]. $x_{i}^{1}$ is the input of the input layer node $i$ and $y_{i}^{1}$ is its output. $y_{j}^{2}$ is the output of the hidden layer node $j . y_{k}^{3}$ is the output of the output layer node $k$. The input layer and the hidden layer are fully and directly connected with no weights.

SVPWM is verified and it can be flexibly implemented on a dedicated IC chip.

In addition to FFNN, another conventional NN structure is radial basis function network (RBFN). In FFNN, the weights of input-to-hidden and hidden-to-output are simultaneously determined. For RBFN, the input layer is directly and fully connected to the hidden layer without weights. The hidden layer is connected to the output layer by weights $W_{j}$, which are the only weight parameters to be determined in the training, as shown in Fig. 9. Typically, the generalization of RBFN is better than FFNN and the training speed and the execution speed are faster. An exemplary application of RBFN in a threephase induction generator to regulate the DC-link voltage and the AC line voltage can be found in [50].

Regarding the number of neurons, there are few principles to determine the optimal number. A generic method is to start with a relatively small number of neurons and then gradually increase it according to the training error. For the activation function in the hidden layer, there are various options, including sigmoid [4], [51], [52], [83], radial basis function [50], [150], hyperbolic tangent function [106], [151], wavelet [46], [53], [84], [152], etc. It is worth mentioning that the wavelet activation function possesses the superior capabilities of convergence speed and generalization.

2) Neural Network with Fuzzy Logic: In control applications, parameter uncertainty and external disturbance should be well considered for system stability and robustness. As a result, an improved variant of NN, i.e., fuzzy neural network (FNN), or neuro-fuzzy system, which is a hybridization of NN and fuzzy logic, is proposed. FNN has the merits from both aspects [100], i.e., the human-like IF-THEN reasoning rules of fuzzy logic that incorporates expert knowledge and cognitive uncertainty, and the strong capabilities of approximation and generalization to any nonlinear systems by the neural network. More theoretical details of FNN can be found in [39].

In [100], an FNN is applied to simulate the sliding-mode control of a boost converter to alleviate the chattering phenom-

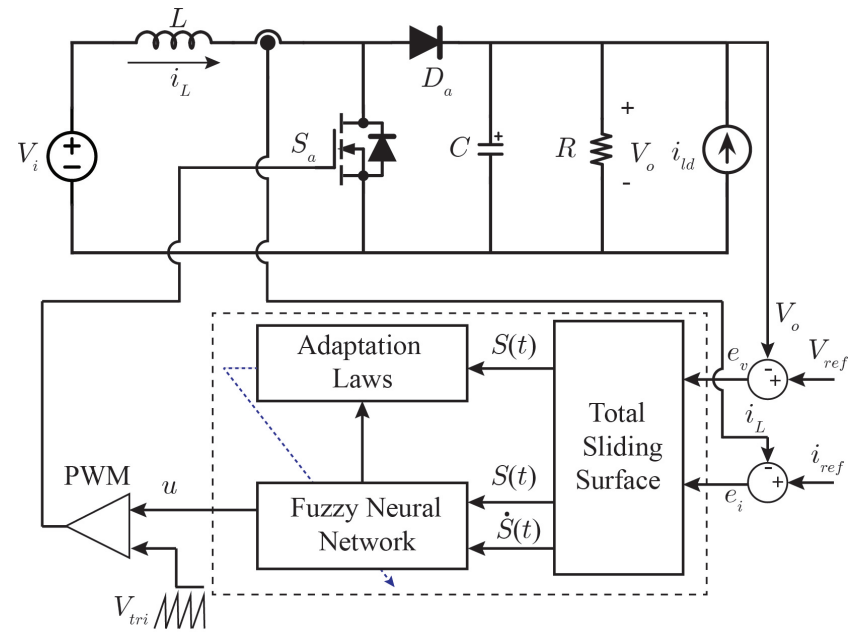

(a) Block diagram of the FNN-based controller for a boost converter.

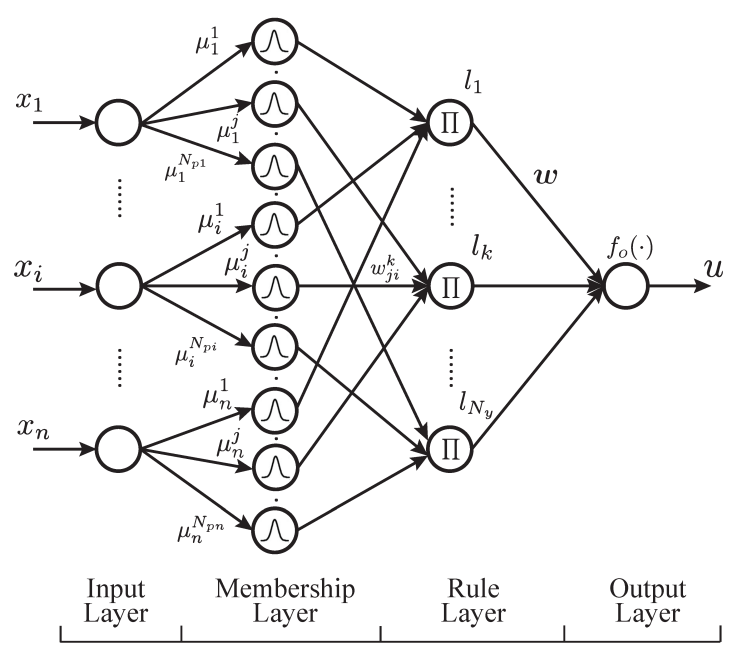

(b) An FNN with a four-layer structure.

Fig. 10. A fuzzy neural network (FNN)-based controller for a boost converter [100]. $x_{1}$ is the sliding surface $S(x)$ and $x_{2}$ is its differentiation, $n=2 . \mu_{i}^{j}$ is the $j^{\text {th }}$ membership function for input $x_{i} . w$ is the weight between layers.

ena. The block diagram of the controller is given in Fig. 10(a) and the FNN structure with four-layer is given in Fig. 10(b). The inputs of the FNN include the sliding surface $S(t)$ and its differentiation $\dot{S}(t)$, which are obtained based on tracking the errors of the average output voltage $e_{v}$ and inductor current $e_{i}$, given the reference voltage $V_{\text {ref }}$ and current $i_{\text {ref }}$. The output control signal is the duty cycle $u$ of PWM. The fuzzy inference is implemented by the rule layer as $l_{k}=\prod_{i=1}^{n} w_{j i}^{k} \mu_{i}^{j}\left(x_{i}\right)$. The network output is obtained as $u=f\left(\sum_{k=1}^{N_{y}} w_{k} l_{k}\right)$. For the voltage control, the voltage tracking performance is evaluated by the mean-square error (MSE) of the output voltage:

$$
\operatorname{MSE}=\frac{1}{T} \sum_{d=1}^{T} e_{v}^{2}(d),
$$

where $T$ is the number of sampling instants. The network tuning aims to reduce the MSE as much as possible to output an accurate and stable voltage. The performance of the FNN can be significantly improved if the membership function is well 


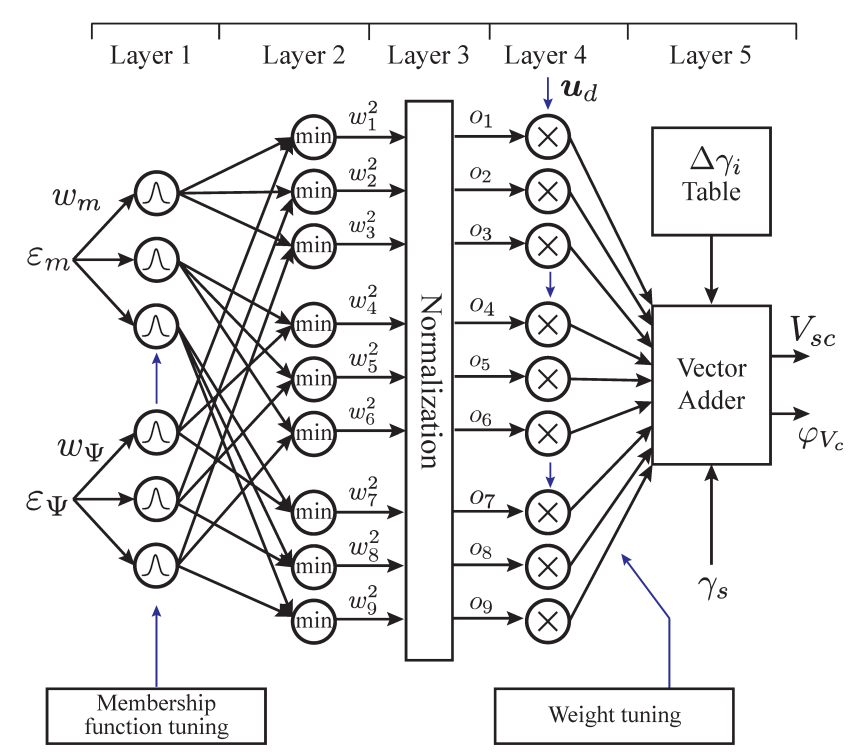

Fig. 11. An adaptive neuro-fuzzy inference system (ANFIS)-based controller for a PWM-inverter-fed induction motor drive [101]. It is a five-layer network structure with the capability of automatic identification of fuzzy rules.

designed. For example, in [46], an asymmetric membership function (AMF) is applied to the controller of a six-phase permanent magnet synchronous motor. It indicates that the learning speed can be improved and the network structure can be simplified compared to conventional membership functions, e.g., Gaussian function [71], [99], [100].

One of the challenges of FNN is the design of the fuzzy rule, where extensive expert experience is usually needed [100]. To overcome this challenge, another typical and effective framework incorporating fuzzy logic and neural network is an adaptive neuro-fuzzy inference system (ANFIS), which can be extended from the four-layer structure in Fig. 10 as a fivelayer topology [101], as shown in Fig. 11. In the ANFIS, the IF-THEN fuzzy rules, which require the involvement of experts, can be generated automatically in the training. For example, in [101], a direct-torque neuro-fuzzy control scheme is developed for a PWM-inverter-fed induction motor drive based on an ANFIS. As shown in Fig. 11, the inputs of the ANFIS-based controller include the flux error $\varepsilon_{m}$ and the torque error $\varepsilon_{\Psi}$. Layer 1 is the membership layer with the input weights $w_{m}$ and $w_{\Psi}$. Layer 2 chooses the minimum from the inputs. Normalization is performed in layer 3. In layer 4, the outputs $o_{i}$ is linearly combined with the network inputs $\mathbf{u}_{d}=\left(\varepsilon_{m}, \varepsilon_{\Psi}\right)$. Layer 5 is the network outputs of the stator voltage command vectors in polar coordinates $V_{c}$ and $\varphi_{V_{c}} \cdot \Delta \gamma_{i}$ is the increment angle and $\gamma_{s}$ is the actual angle of the stator flux vector. In contrast to the conventional training schemes, the parameter tuning of the ANFIS is completed interactively with the backpropagation algorithms (for membership functions) and the least square method (for parameters in $4^{\text {th }}$ layer). More theoretical details of the training methods of the ANFIS can be found in [153].

3) Neural Network with Recurrent Units: The NN structures in Section IV-B1 and FNN in IV-B2, however, are only applicable to the static relationship mapping and behavior

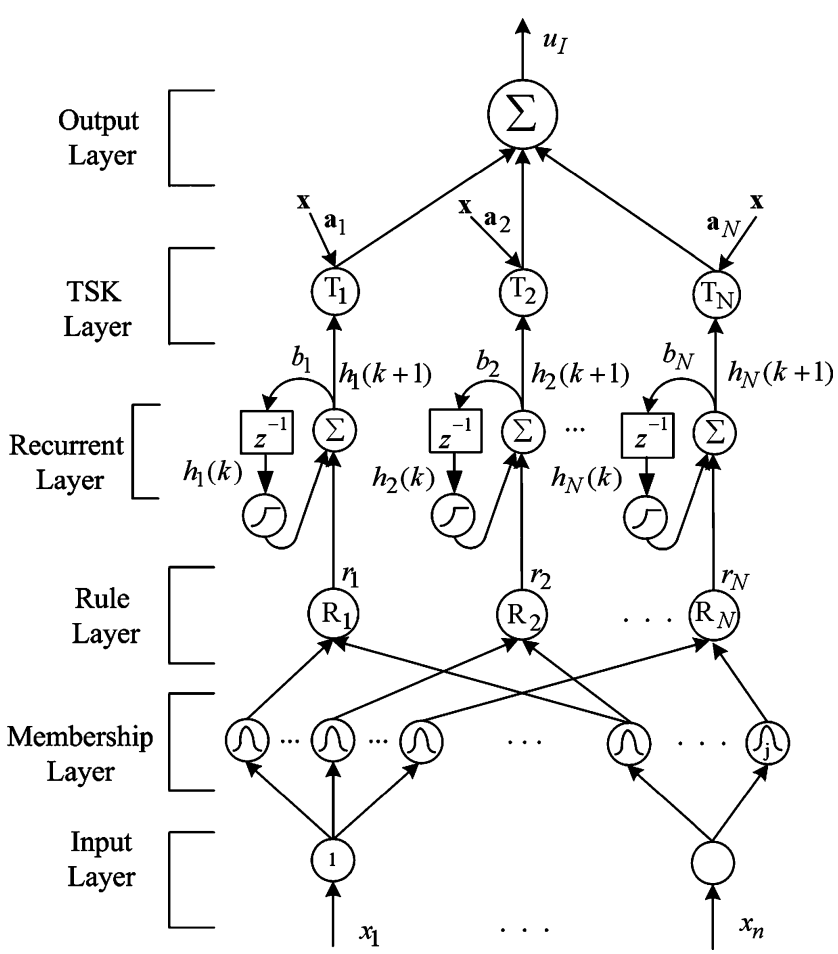

Fig. 12. A recurrent fuzzy neural network (RFNN) controller for the highprecision trajectory tracking control of a linear microstepping motor driver [99]. A memory unit of time-delayed feedback connection $Z^{-1}$ is added to enable the dynamic capability of neural network controller.

characterization. The dynamic performance of the controller is critical for the transient response. To enable the dynamic capability of the neural network controller, a memory unit of time-delayed feedback connection $Z^{-1}$ is usually inserted to formulate recurrent neural network (RNN) [107], as shown in Fig. 12. The outputs of the network not only depend on the present inputs but also on the previous ones. As a result, the network structure can tackle the time series data to facilitate the better performance of dynamics and sensitivity.

In [106], a robust controller based on RNN is proposed for single-phase grid-connected converters for better control performance in the presence of system parameter changes. The training of the RNN is completed by the LevenbergMarquardt (LM) method [13], [82], [106]. The harmonics can be significantly reduced by using the proposed RNNbased controller, and the requirements of the high sampling and switching frequency and the damping policies for the conventional control methods can be mitigated. A similar RNN structure, which is also termed as Elman neural network (ENN), can be found in [52].

In addition to the performance of dynamics, fuzzy logic is also incorporated into RNN in order to improve the performance of robustness. For example, in [99], a controller based on a TSK-type self-organizing recurrent fuzzy neural network (RFNN) is proposed for a high-precision trajectory tracking control of a linear microstepping motor driver. The network structure is given in Fig. 12. The TSK-type self-organizing RFNN is applied to model the inverse dynamics of the driver. Compared to the FNN in Fig. 10(b), the key of the RFNN is the insertion of a recurrent layer, where the delayed neuron 
output $h_{i}(k)$ is returned as the neuron input to facilitate the network dynamics. The network diagram and size are adjusted by the self-organizing method, and the respective network parameters are tuned with the method of recursive least square. As a result, the network diagram and its parameters can be optimized simultaneously.

4) Training Methods of Neural Network: Essentially, the training of the neural network is an optimization task. Of course, it can be completed with conventional optimization methods, e.g., PSO [51], recursive least square [99], Kalman filter [105], etc. Considering a large number of parameters in the neural network, these conventional optimization methods are generally inefficient. As a result, an elaborate training scheme is developed, i.e., backpropagation algorithm [4], [50], [52], [53], [71], [83], [84], [150]. More theoretical details of the backpropagation algorithm can be found in Chapter 5 of [1].

The backpropagation algorithm is based on the idea of steepest gradient descent. One of the key steps in the backpropagation algorithm is the iteration of the weight update:

$$
w_{k+1}=w_{k}-\eta_{k} g_{k}
$$

where $w_{k}$ is the current weight, $g_{k}$ is the current gradient, $\eta_{k}$ is the learning rate, and $w_{k+1}$ is the weight of the next iteration. To calculate the gradient $g_{k}$ and find the steepest direction of gradient descent efficiently, various improved variants of the backpropagation algorithm have been proposed, e.g., Levenberg-Marquardt method [13], [82], [106], resilient backpropagation algorithm, conjugate gradient algorithm, onestep secant algorithm, etc. Note that it is challenging to determine the most suitable training algorithm for a specific task. It depends on multiple factors, including problem complexity, dataset size, number of parameters, task types of classification or regression, etc. A useful reference can be found in Matlab Manual of Neural Network Toolbox [40], where the theoretical details, advantages, limitations, and comparisons of these training algorithms are thoroughly analyzed with several benchmark examples. It is worth mentioning that LevenbergMarquardt method is one of the most widely used methods for the applications in power electronics with a fast convergence speed and a high accuracy.

Considering whether the training dataset is available in a batch form or in a sequential form, the training scheme of the neural network can be completed in either batch learning, which is also termed as offline learning, or sequential learning, which is also termed as online learning or incremental learning.

For batch learning, the gradient $g_{k}$ in (4) is calculated based on all the data points in the dataset for the parameter updates. It generally applies to the case where the whole dataset is available before the neural network is implemented for field application, e.g., the waveform processing and delayless filtering in [82].

For sequential learning, the gradient $g_{k}$ in (4) is calculated based on every newly available data point or several newly available data points forming a mini-batch. Therefore, the

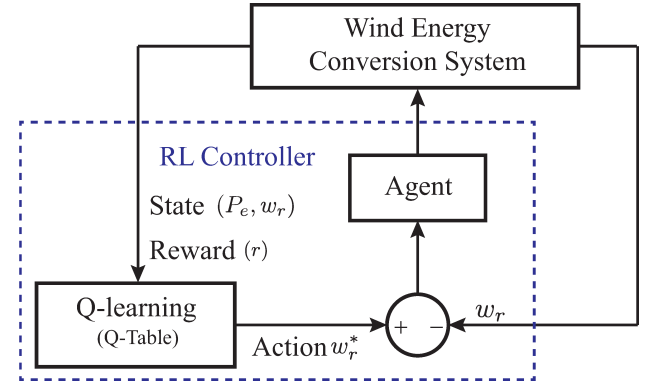

Fig. 13. Framework of reinforcement learning in the maximum power point tracking (MPPT) controller of wind energy conversion systems [5], [138]. A Q-table is formulated to save the optimal generator rotor speed $w_{r}^{*}$ to be performed given the current system state $s_{t}$, including the current electrical output power $P_{e}$ and the generator rotor speed $w_{r}$.

learning process is incrementally completed. This feature is especially useful for the case where the training data can only be sequentially obtained in field application. The intelligent controller [53] is a typical case of a sequential training scheme since the input data of the neural network can only be available sequentially by interacting with the output of the control command and the system. With this adaptive capability, the neural network can be re-parameterized and reconfigured for tracking the system parameter shifts. One of the key steps for the sequential learning is determining a suitable learning rate $\eta_{k}$ in (4), since a larger $\eta_{k}$ will result in system instability and a smaller $\eta_{k}$ will lead to slow convergence. The optimal learning rate $\eta_{k}$ can be determined by using the metaheuristic methods in the training, e.g., PSO in [50], [52], [53] and differential evolutionary in [46]. As a result, the sequential learning process can be stable and converges fast.

\section{Reinforcement Learning-based Controller}

With reinforcement learning, the controller learns a goaloriented control strategy by interacting with the physical system or its simulation model [138]. It accumulates experience progressively and learns a specific control strategy that maximizes predefined goals.

One of the relevant applications of RL-based controller is the MPPT in renewable energy systems [5], as shown in Fig. 13. Specifically, a real-time intelligent MPPT algorithm using $\mathrm{RL}$ is proposed for a wind energy conversion system. With the online learning capability of RL by interacting with the environment, an optimum control strategy is formulated in the Q-table. The Q-table consists of elements of state transition probability $q\left(s_{t}, a_{t}\right)$, which can facilitate the maximized power output (or reward) if action $a_{t}$, i.e., the expected generator rotor speed $w_{r}^{*}$, is performed given the current system state $s_{t}$, including the current electrical output power $P_{e}$ and the generator rotor speed $w_{r}$. As a highlight, the wind turbine parameter and the wind speed are not required. This work is further extended by integrating an NN into the Q-learning of RL [6]. In this way, the challenges in the determination of the state space are avoided. The online learning process can be reactivated once the learned optimal relationship is destructed by the system aging behaviors. It significantly improves the autonomous capability of the wind energy conversion system. 
Table IV: The advantages and limitations of AI algorithms in control applications. FFNN - Feed-forward Neural network and its variants, FNN - Fuzzy neural network and its variants, RNN - Recurrent neural network and its variants, RFNN - Recurrent fuzzy neural network and its variants. Superior: +++ , intermediate: ++ , inferior: +

\begin{tabular}{|c|c|c|c|c|c|c|}
\hline \multirow{2}{*}{ Performance } & \multirow{2}{*}{$\begin{array}{l}\text { Fuzzy } \\
\text { logic }\end{array}$} & \multicolumn{4}{|c|}{ Machine learning } & \multirow{2}{*}{$\begin{array}{l}\text { Reinforcement } \\
\text { learning }\end{array}$} \\
\hline & & FFNN & FNN & RNN & RFNN & \\
\hline $\begin{array}{l}\text { Approximate } \\
\text { capability }\end{array}$ & + & ++ & +++ & +++ & +++ & +++ \\
\hline Robustness & ++ & + & +++ & ++ & +++ & +++ \\
\hline $\begin{array}{l}\text { Computational } \\
\text { burden }\end{array}$ & +++ & +++ & ++ & + & + & + \\
\hline $\begin{array}{l}\text { Dataset } \\
\text { requirement }\end{array}$ & ++ & +++ & ++ & + & + & N/A \\
\hline Dynamics & No & No & No & Yes & Yes & Yes \\
\hline $\begin{array}{l}\text { Expert } \\
\text { knowledge } \\
\text { embedded } \\
\text { capability }\end{array}$ & Yes & No & Yes & No & Yes & No \\
\hline
\end{tabular}

A similar example can be found in [140], where RL is applied to the MPPT control of a buck converter of photovoltaic arrays.

For the neural network-based controller, the learning process is completed from examples provided by an external supervisor. While the RL controller can learn the experience by directly interacting with the environment through actions and rewards. It is worth mentioning that the training of the RL controller is based on the interactions between the controller and the system, and the offline dataset is unnecessary in this case. As a result, the RL-based controller is beneficial to new systems without existing datasets.

\section{Discussions}

A summary of the advantages and limitations of AI algorithms in control applications is given in Table IV. It is worth mentioning that the dynamic performance, robustness, generalization, and convergence speed of AI algorithms are critical in control applications. The algorithm complexity and computational burden are the major challenges. Thus, high-performance Digital Signal Processor (DSP) or Field Programmable Gate Array (FPGA) is necessary for practical implementations.

\section{Maintenance}

Although reliability characteristics have been elaborately considered in design and control, power electronic systems still undertake various risks and even catastrophic failures due to complex and severe working environments [18], [154], [155]. The reliability and safety of power electronic components, converters, and systems are of great importance for field applications. In maintenance, preventive activities, including condition monitoring, anomaly detection, fault diagnosis, RUL prediction, etc., are effective approaches to ensure that intended functions can be properly executed. These activities are aligned with the IEEE standard framework of PHM for electronic systems [156]. Fig. 14 presents a systematic flowchart of maintenance activities in power electronic systems. Generally, it consists of three parts:
1) Offline training and knowledge learning: It integrates various aspects of knowledge including historical monitoring data, simulation data, accelerated aging test experiment, failure mode and effects analysis (FMEA), etc. Moreover, ensemble methods or fusion techniques are typically applied to this part for performance improvement. As a result, physical system dynamics and behaviors (e.g., degradation behavior) can be accurately characterized as offline models based on the information of the unit population.

2) Condition monitoring and health assessment: This part deals with the health assessment of the unit in service subjected to the online condition monitoring in field applications. The offline model is tailored and individualized to the unit in service through the model parameter tuning layer by adapting to field operational environment and workload. The functions include the noninvasive parameter identification, data preprocessing (e.g., data cleaning), feature mining, anomaly detection, fault diagnosis, and RUL prediction. In this way, insightful knowledge for decision-making can be extracted from the continuous condition monitoring information.

3) Management and decision-making: In this part, the supportive knowledge of health assessment are returned for optimal decision making. With this feedback, control policies (e.g., power routing) can be adjusted to maximize the system performance given the real-time health status. Moreover, economical maintenance policy can be made to facilitate the condition-based and predictive maintenance.

Subsequently, the relevant applications of AI in maintenance in terms of these three parts are discussed in detail.

\section{A. Condition Monitoring}

Condition monitoring [20], [157], [158] in power electronics includes system parameter identification, data preprocessing, and feature mining. The condition monitoring information is applied to uncover hidden and informative insights, which serve as a basis for the subsequent PHM applications.

1) System Parameter Identification: The system parameter identification [159] deals with information acquisition for critical components. Developing specific hardware for parameter identification (e.g., temperature-sensitive electrical parameters of IGBTs [158]), however, is quite a challenging task due to features of power electronic systems, e.g., very tight space in a power module, very fast switching frequency, relatively insignificant parameter changes in terms of aging [157], etc. One of the promising solutions is noninvasive method without any extra hardware implementation, where information of interest can be inferred or estimated indirectly from available physical signals. As a result, the condition monitoring can be implemented with a sensorless and cost-efficient solution, which is favorable for industrial practitioners. Generally, the system parameter identification can be categorized into modelfree and model-based methods, considering whether the system dynamics and models are required. 


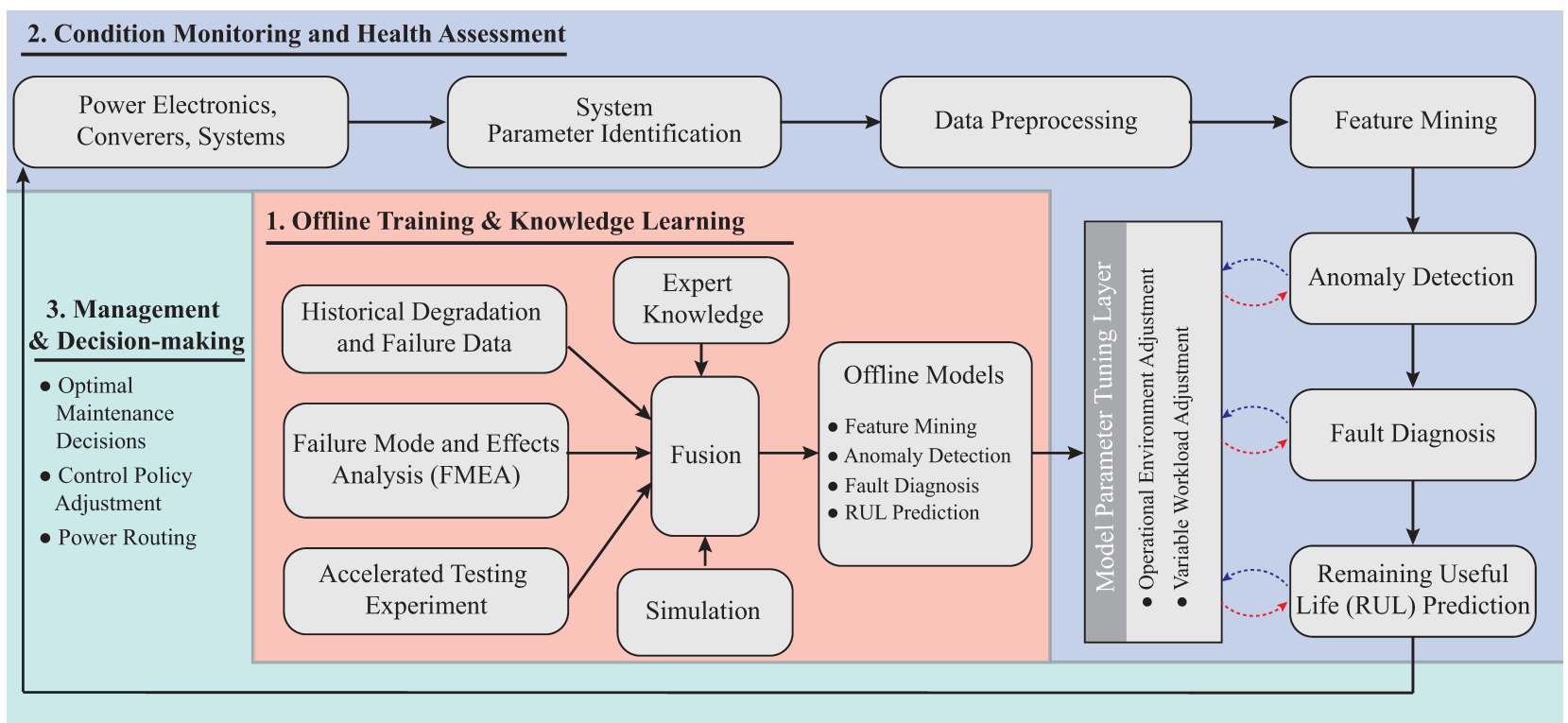

Fig. 14. Flowchart of maintenance in power electronic systems.

For the model-free method, no prior knowledge of the system dynamics is required. Essentially, it deals with the regression capability of $\mathrm{AI}$ algorithms to construct a relationship between the inputs and outputs. For example, in a three-phase front-end diode bridge motor drive, the current $i_{\mathrm{a} \text {,out }}$ in $a$-phase and the DC-link ripple voltage $\Delta v_{\mathrm{dc}}$ are considered as the inputs, and the capacitance $C$ is applied as the output for the training of an FFNN [86]-[88]. In this way, the relationship between the input signals and the capacitance is established and thus the capacitance can be inferred indirectly. Similarly, it is demonstrated that the capacitance can be estimated by the FFNN constructed by the frequency domain information of DC-link voltage ripple. The potentials of FFNN in the capacitance estimation are illustrated in a hardware prototype [88].

In [108], considering the dynamic capability of RNN, an impedance identification method is proposed based on RNN to enable the stability analysis for power electronic systems over a wide frequency range. The RNN is applied to build a model that can produce identical outputs as the physical system given the same inputs. The inputs of RNN include three-phase voltages $v_{a}, v_{b}, v_{c}$. The output is the $a$-phase current $i_{a}$. As a result, the RNN-based model possesses the same frequency characteristics as the physical one. It can be performed for the impedance identification without interrupting the system operation.

In [103], an improved ANFIS is applied to estimate the capacitance and equivalent series resistance (ESR) of the supercapacitor. At monitoring time $t$, the inputs of the ANFIS include the supply voltage $V_{t}$, the supercapacitor temperature $\theta_{t}$, and a time series $\mathrm{ESR}_{t-400: 100: t}$ consisting of 5 previous ESR data points. The output of the ANFIS is the ESR estimations in future $p$ steps. Experimental analysis indicates that ESR of supercapacitor can be accurately estimated and the normalized root mean square error of the ESR estimation is as small as 0.025 at condition monitoring time of $2600 \mathrm{~h}$.

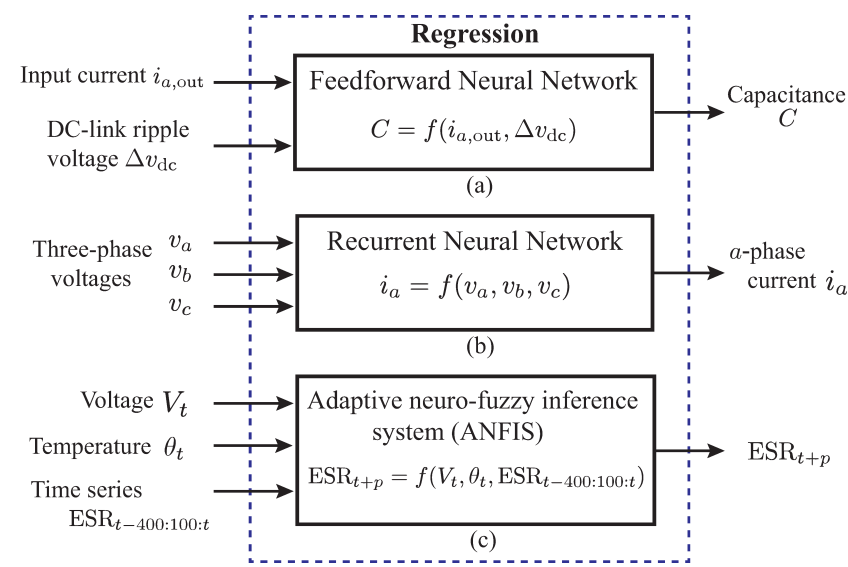

Fig. 15. Examples of model-free methods of system parameter identification with AI. (a) Capacitance identification of DC-link capacitor [88]; (b) $a$ phase current estimation for calculating the impedance measurement of power electronic system [108]; and (c) Equivalent series resistance (ESR) estimation in future $p$ steps for supercapacitors [103].

A summary for the framework of model-free parameter identification methods is given in Fig. 15. It can be seen that AI methods serve as the regression tool $f(\cdot)$ between the available input signals and the parameter to be monitored.

The model-free method is attractive for industrial applications due to less hardware cost. However, it is typically sensitive to external noise and disturbance due to the lack of system model. Thus, its robustness should be carefully considered. This issue can be possibly mitigated with a large amount of data in the training stage [159] to cover situations in field applications as much as possible. Nevertheless, the data collection is time-consuming and costly.

Another category of the system parameter identification is the model-based method. As the name implies, for a modelbased method, system physics and models are partially known in advance and the identification model is formulated with unknown model parameters. In this way, the system identification 


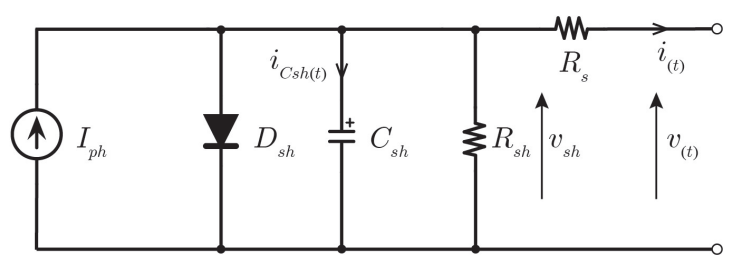

Fig. 16. Dynamic model of a PV panel for parameter identification with model-based method [69]. System parameters includes the input current $I_{p h}$, output current $I_{o}\left(i_{(t)}\right)$, voltage $v_{s h}$ across capacitor $C_{s h}$, resistor $R_{s h}$, p-n junction capacitance $C_{s h}$, and resistor $R_{s}$.

task is equivalent to the exploration of optimal parameters of the model, which is essentially an optimization task. In this case, AI, especially the metaheuristic methods, is utilized as an optimizer to find the optimal solutions. Numerous approaches such as PSO [57], crow search algorithm [73], GA [69], etc, or their improved variants, can be exploited.

In [69], a parameter identification method for the health diagnostic of a PV panel is developed. The equivalent circuit of the PV panel is given in Fig. 16, and its system model is explicitly derived as

$$
\left\{\begin{array}{l}
i_{C s h}\left(v_{s h}, v\right)=I_{p h}-i_{D}\left(v_{s h}\right)-\frac{v_{s h}}{R_{s h}}-\frac{v_{s h}-v}{R_{s}}, \\
\frac{d v_{s h}\left(v_{s h}, v\right)}{d t}=\frac{1}{C_{s h}}\left[I_{p h}-i_{D}\left(v_{s h}\right)-\frac{v_{s h}}{R_{s h}}-\frac{v_{s h}-v}{R_{s}}\right]
\end{array}\right.
$$

where $I_{p h}$ is the input current, $I_{o}$ is the output current, $v_{s h}$ is the voltage across the capacitor $C_{s h}, R_{s h}$ is the resistance, and $C_{s h}$ is the p-n junction capacitance. As a result, the parameter identification is equivalent to find a parameter set $G=\left\{I_{p h}, I_{o}, v_{s h}, R_{s h}, C_{s h}, R_{s}\right\}$ that ensures an identical output as the physical system. By injecting large signal disturbances to the panel voltages in the testing stage, the dynamic response of the current-voltage characteristics is sampled to calculate the objective function as

$$
f_{o b j}(G)=\frac{1}{N_{1}-N_{2}+1} \sum_{k=N_{1}}^{N_{2}}\left(i_{p}[k]-i[k]\right)^{2},
$$

where $i_{p}[k]$ and $i[k]$ are the current output of the model and the physical system, respectively, and $N_{1}$ and $N_{2}$ are the start index and the end index for the sampling. Subsequently, an improved GA method is used to explore an optimal solution minimizing $f_{o b j}(G)$ in (6). A similar investigation can be found in [57], where a modified PSO algorithm is applied to the internal parameter identification of a PV panel.

Due to the involvement of system dynamics and models, the amount of data required for the estimation can be significantly reduced for the model-based methods. Also, the overfitting risk in the model-free methods can be mitigated. It exhibits better dynamics to handle unexpected disturbance and switchable working modes. However, due to the system complexity, the system dynamics and models are challenging to formulate in most cases.

For parameter identification methods in power electronics, the accuracy and robustness under the complex environment

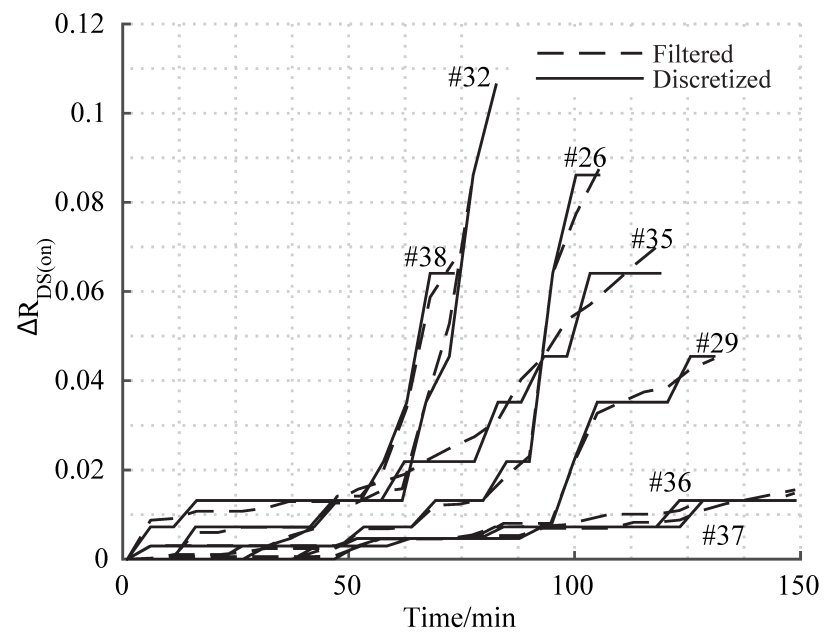

Fig. 17. $k$-means clustering method for discretization of filtered degradation paths of increment of drain-to-source on-state resistance $\Delta R_{\mathrm{DS} \text { (on) }}$ of different power MOSFET devices \#26, \#29, \#32, \#35, \#36, \#37, \#38 [131].

should be considered. For example, for the condition monitoring of power MOSFETs in [131], the device is considered as failed if there is an increase of $0.08 \Omega$ for the degradation indicator of drain-to-source on-state resistance $R_{\mathrm{DS}(\text { on) }}$. Such a tiny increment is challenging to observe. Thus, more research efforts are necessary to improve the sensitivity of the AI-based parameter identification methods. Moreover, it is worth mentioning that computational burden and embedded capabilities should be considered for field applications.

2) Data Preprocessing and Feature Mining: Data preprocessing and feature mining are concerned with refining the raw data to better serve the applications, e.g., fault diagnosis. By exploring dataset structure, it includes data cleaning to reduce noise, data clustering to discover groups of similar data points, density estimation to identify the data distribution, data compression that projects high-dimensional data down to low-dimensional data to reduce the number of features, data fusion to integrate multiple information sources, etc. Typically, the performance of the subsequent PHM application, e.g., the diagnostic accuracy, can be significantly improved if the data preprocessing and feature mining are properly conducted.

In [131], a reliability assessment method for power MOSFETs based on a continuous-time Markov chain is proposed. To discretize the continuous degradation path of power MOSFETs without breaking the inherent monotonicity, a $k$-means method is applied to divide the evolution of drain-to-source on-state resistance $R_{\mathrm{DS}(\mathrm{ON})}$ into 11 discrete states, as shown in Fig. 17.

In [133], a health state identification method for IGBTs based on self-organizing maps (SOMs) is proposed. It is essentially a clustering task. The states of the device are clustered as the healthy state, partially degraded state, heavily degraded state, and failure state, considering the distance between the input measurements (including collector current $I_{c}$, collector-emitter voltage $V_{c e}$, and case temperature $T$ ) and the best matching unit of the trained SOMs.

In [160], a composite failure precursor of SiC MOSFETs is developed with a data fusion technique of genetic pro- 
gramming, which is a variant of GA. It integrates multiple degradation signals of a power semiconductor device in a nonlinear way. Since the composite failure precursor is directly optimized in terms of the RUL prediction model, the prediction accuracy is improved by $35.3 \%$ and the prediction uncertainty is reduced by $16.3 \%$. It indicates that data fusion in condition monitoring is potentially useful especially for systemlevel applications (e.g., converters), where multiple physical degradation signals exist.

An integrated toolbox "Diagnostic Feature Designer" for the feature identification is available in Matlab [161], which can be applied to the data preprocessing and feature mining as an automatic tool.

\section{B. Anomaly Detection and Fault Diagnosis}

The anomaly detection makes a binary decision and focuses on the abnormal behavior identification. It provides an indication when the rated system characteristics or nominal parameters exceed the predefined safety range. Once the anomaly behavior occurs, the fault diagnosis [19] identifies and locates the detailed failure modes subsequently. Essentially, anomaly detection and fault diagnosis are the classification, regression, or clustering tasks. Based on the learned relationship from the training stage, it determines the fault label when a new fault signature becomes available. Note that the feasibility of AI-based anomaly detection and fault diagnosis is based on two assumptions [33]: firstly, the fault occurrence in any components has an impact on the fault signature; secondly, the impact on these signatures varies with different fault modes and fault locations. The methods of anomaly detection and fault diagnosis can be categorized as supervised learning methods and unsupervised methods.

1) Supervised Learning Methods: In [93], an FFNN is applied to establish the nonlinear relationship of the inputs and outputs of a full-bridge diode rectifier. The training of the FFNN is completed at the normal operation mode of the rectifier, as shown in Fig. 18. As a result, the principles and mapping relationship between the inputs, including input voltage $v_{i}(t)$, input current $i_{i}(t)$, and output current $i_{o}(t)$, and the output signal of output voltage $v_{o}(t)$ are characterized, considered as a digital emulator indicating the normal operational mode of the rectifier. This digital emulator and the physical rectifier are simultaneously operated and their outputs are compared in real-time. Once the monitored output voltage of physical rectifier significantly deviates from the output of FFNN, it suggests that the rectifier runs into an abnormal mode, which facilitates the anomaly detection. In this case, the FFNN essentially serves as the regression tool.

In [90], an open-circuit fault diagnosis algorithm is proposed for the inverter in a microgrid system subjected to varying load conditions. A signal processing method is proposed to reduce the required amount of information for the fault representation and suppress the impact of the load change. An FFNN is used as a diagnostic classifier. The computational burden of the proposed method can be reduced to $10 \%$ of that of the existing algorithms. In this case, the FFNN serves as a classification tool. Similar fault diagnosis ideas include the

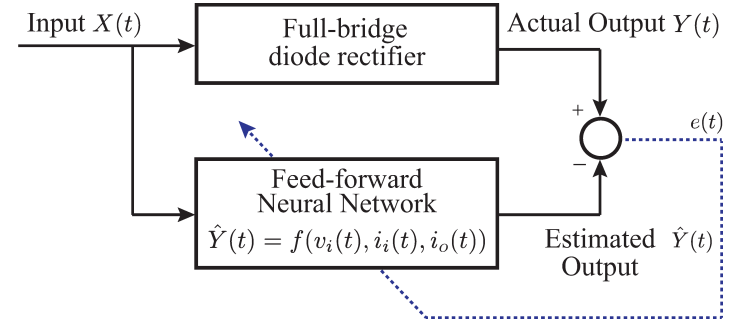

Fig. 18. Feed-forward neural network for anomaly detection of a full-bridge diode rectifier. The inputs of the neural network include input voltage $v_{i}(t)$, input current $i_{i}(t)$, and output current $i_{o}(t)$; the output of the neural network is output voltage $v_{o}(t)$ [93]

ANFIS to determine the severity levels of a capacitor in the DC-link filter [102].

In [112], a multi-switches fault diagnosis algorithm for voltage-source inverters is proposed. An echo state network (ESN) is used as a diagnostic classifier given small lowfrequency data. Note that ESN is an improved variant of RNN to avoid gradient exploding and vanishing in the training. In this work, the diagnostic performance of ESN is compared with the FFNN, the FFNN with a wavelet activation function, and the RBFN. It indicates that the ESN is superior in the sensitivity, design process, and training speed.

In [115], an 1-D convolutional neural network (CNN) is applied to the fault diagnosis of a modular multilevel converter. One advantage of 1-D CNN is that the feature extraction and diagnostic classification can be integrated together, which enables the fault diagnostics on the raw data directly. In this way, the feature extraction, which is usually experienceintensive, can be avoided. The experimental results indicate that the proposed method is highly reliable and provides a detection accuracy of $98.9 \%$ and a fault diagnostic accuracy of $99.7 \%$ within $100 \mathrm{~ms}$.

In addition to the above neural network-based methods, kernel methods, including the support vector machine and the relevance vector machine, are also applied for anomaly detection and fault diagnosis. One advantage of the kernel methods is that the dataset size requirement is relatively lower than the neural network-based methods.

In [7], based on the time-domain fault features, a support vector machine-based fault diagnosis method is proposed for incipient yet progressive faults of IGBTs in an inverter. The training of SVM can be completed by metaheuristic methods (e.g., PSO, GA, etc.). For a total of 41 fault classes, it achieves an average accuracy of $94.82 \%$ being robust to both load variations and motor parameter shifts.

In [127], a relevance vector machine (RVM) is applied for the fault diagnosis of a cascaded H-bridge multilevel inverter. Principal component analysis (PCA) is applied to extract the fault signal feature. Experimental analysis indicates that the RVM outperforms the FFNN and the SVM, with $100 \%$ diagnostic accuracy in this specific case study. Compared to SVM with the direct fault label as its output, RVM is formulated under the Bayesian framework. It makes probabilistic outputs of the fault information, which possesses good theoretical guidance and is favorable to the uncertainty analysis on diagnostic results. Generally, for the same task, 
the RVM is sparser than SVM, indicating faster speed for field applications. However, the training time of RVM is generally longer than SVM.

2) Unsupervised Learning Methods: In [136], principal component analysis (PCA) is applied to the anomaly detection of SiC MOSFETs. Multiple statistical features, including kurtosis, skewness, etc., are considered as the inputs of the PCA algorithm. The output is compact with fewer features and a transformation matrix. For field applications, the newly available data is applied to the transformation matrix for the calculation of an anomaly index. Abnormal behavior is notified when the anomaly index exceeds a predefined threshold. The method is verified by a processor-in-the-loop experiment. This detection mechanism is similar to [93]. Other unsupervised learning methods in anomaly detection and fault diagnosis, including $k$-means and SOMs, can be found in [118].

3) Discussions: Table V summarizes the features of typical AI algorithms and their variants for anomaly detection and fault diagnosis. It can be seen that each AI algorithm possesses advantages and limitations. To fully exploit the advantages of each algorithm, it is effective to combine multiple algorithms for a decision-level fusion to improve the diagnostic accuracy and robustness. An example of decision-level fusion for fault diagnosis of IGBTs can be found in [96]. More ensemble methods to combine multiple algorithms can be found in Chapter 14 in [1]. From the AI perspective, there is a negligible difference between power electronics and other engineering areas (e.g., electromechanical applications) in terms of the anomaly detection and fault diagnosis tasks. Two reviews of AI methods in anomaly detection and fault diagnosis can be found in [162], [163].

Note that various AI methods and their variants have been successfully applied to anomaly detection and fault diagnosis. There are differences in terms of how the data are collected and types of available data in different applications, which is an important aspect of practical applications of AI. An integrated platform "Predictive Maintenance Toolbox" is available in Matlab [164], which includes various algorithms of anomaly detection and diagnostics. It is beneficial for the method development and benchmark analysis. From the AI perspective, most of the methods can be interchangeably applied with a comparable performance in terms of the evaluation accuracy. Although the accuracy can be further improved by advanced algorithms (e.g., deep learning methods), the accuracy improvement after a high score, e.g., 90\%, is relatively less significant compared with other practical concerns. More considerations should be devoted to the gap between theoretical algorithms and practical implementations, where the practical considerations include

1) In addition to the single component fault, the failure mode of multiple components failed simultaneously should be considered. The dependence and coupling effects among the component failures should be incorporated into the diagnostic algorithms.

2) Considering the challenges in the data acquisition of power electronic systems, the training dataset for practical application is typically limited. This situation is even worse for a dataset with unbalanced fault labels, i.e., the

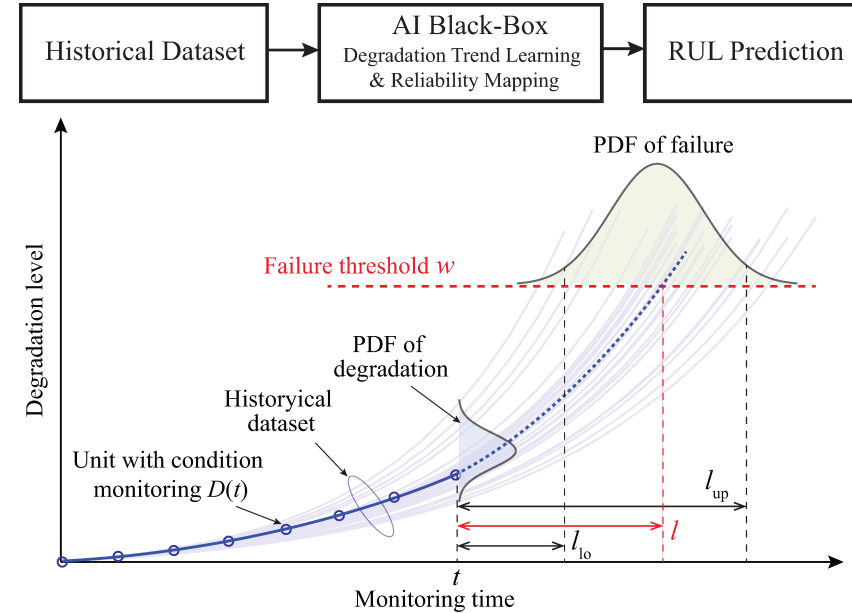

Fig. 19. Flowchart and procedures of AI methods for remaining useful life (RUL) prediction of power electronic systems. PDF: probability density function.

ample data of the normal operation case and the scarcity of data with fault labels due to catastrophic failures. Thus, the algorithm applicability given limited size of dataset and poor quality dataset should be investigated.

3) The practicality, including computational burden, adaptive capability, robustness, difficulty of algorithm design and debugging [112], implementation cost, etc, should also be comprehensively considered.

\section{Remaining Useful Life Prediction}

Lifetime prediction in the design phase is to support the DfR, which refers to the feature of a population of units. As one of the critical aspects of Prognostics and Health Management [165], the RUL prediction is not to predict the lifetime of a population of units. It predicts the residual lifetime of an individual unit in service based on the condition monitoring information. There are associated uncertainties in the lifetime prediction, including model calibration errors, manufacturing tolerances, variations of operational environments and workload, etc. These uncertainties result in inaccurate reliability estimates for a specific unit in field operation [166]. RUL prediction is applied as an additional tool to reduce the uncertainties for reliability-critical, safety-critical, or availabilitycritical applications.

The flowchart and procedures for RUL prediction are given in Fig. 19. The regression model can be established based on historical dataset. The probability density function (PDF) of degradation level at any specific condition monitoring time can be estimated based on the regression model. The PDF of the RUL can be derived from the PDF of the degradation level. Given the fact that the system is properly functioning at condition monitoring time $t$, its RUL $l$ is defined as the residual lifetime when the degradation process $D(t)$ exceeds the failure threshold $w$, i.e.,

$$
l=\inf \left\{l: D(t+l) \geq w \mid D(t)<w, \mathbf{D}_{1: j}\right\},
$$

where $\mathbf{D}_{1: j}$ is the cumulative $\mathrm{CM}$ information up to time $t$. Note that RUL $l$ is a random variable. In addition to its 
Table V: Algorithm comparisons of anomaly detection and fault diagnosis in power electronic systems. N/A: not available

\begin{tabular}{|r|l|l|l|l|l|}
\hline References & Methods & Number of faults & Speed & Accuracy & Advantages \& Limitations \\
\hline$[94]$ & $\begin{array}{l}\text { Feed-forward neural } \\
\text { network }\end{array}$ & 5 & N/A & $>85.0 \%$ & $\begin{array}{l}- \text { Training may result in a local solution } \\
- \text { Sensitive to weights }\end{array}$ \\
\hline$[112]$ & Echo state network & 4 & - Training time: $0.0626 \mathrm{~s}$ & N/A & $\begin{array}{l}- \text { Training solution is globally optimal } \\
- \text { Less sensitive to weights } \\
- \text { Fast training speed }\end{array}$ \\
\hline$[115]$ & $\begin{array}{l}\text { 1-D convolutional } \\
\text { neural network }\end{array}$ & 9 & - Testing time: $<0.1 \mathrm{~s}$ & $99.70 \%$ & - Feature mining is unnecessary \\
\hline$[7]$ & Support vector machine & 41 & N/A & $94.82 \%$ & - Lower requirement for feature mining \\
\hline$[127]$ & $\begin{array}{l}\text { Relevance vector } \\
\text { machine }\end{array}$ & 33 & - Testing time: $<0.14 \mathrm{~s}$ & $>97.30 \%$ & $\begin{array}{l}- \text { Probabilistic outputs } \\
\text { - Higher model sparsity and speed }\end{array}$ \\
\hline$[117]$ & Bayesian network & 22 & - Training time: $0.172 \mathrm{~s}$ & $98.99 \%$ & $\begin{array}{l}\text { - Probabilistic outputs } \\
- \text { Better toleration for sensor noise and bias }\end{array}$ \\
\hline$[33]$ & Fuzzy logic & 12 & - Testing time: $<0.017 \mathrm{~s}$ & N/A & - Fast testing speed \\
\hline$[96]$ & Ensemble model & 3 & N/A & $>97.0 \%$ & - Better diagnostic accuracy and robustness \\
\hline
\end{tabular}

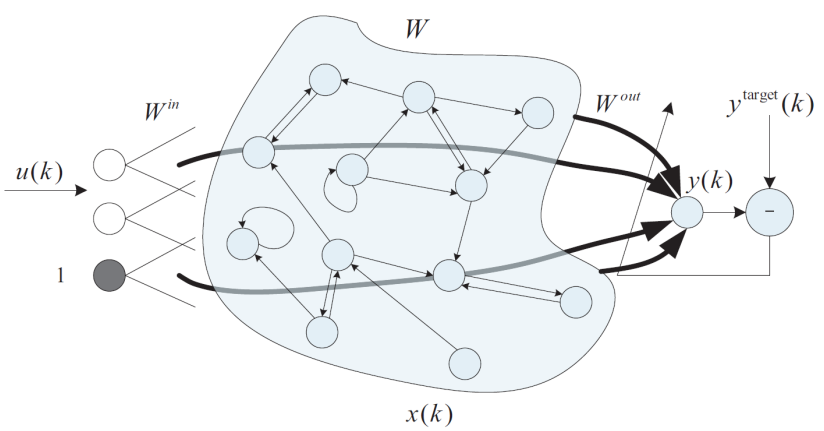

Fig. 20. Remaining useful life prediction of power MOSFETs based on echo state network [111]. For the network training, the input weights $W^{\text {in }}$ and the recurrent weights $W$ are randomly generated. The output weights $W^{\text {out }}$ are estimated by least-square methods.

expected value, the uncertainty metrics with the lower and upper confidence interval $\left(l_{l o}, l_{\text {up }}\right)$ are also of great importance. AI methods in RUL prediction is typically dealing with a nonlinear regression between the degradation information and the corresponding RUL based on the training dataset [167]. In this way, degradation patterns can be characterized. Once the degradation patterns have been learned, it can be directly projected based on the regression model to facilitate the future degradation level prediction. As a result, the RUL can be estimated.

In [111], an echo state network is applied to the RUL prediction of power MOSFETs, as shown in Fig. 20. The input of the echo state network is the degradation indicator drain-tosource on-state resistance $R_{\mathrm{DS} \text {,(on) }}$ at times $k-1$ and $k$, and the output is the $R_{\mathrm{DS} \text {,(on) }}$ at time $k+1$. To facilitate the adaptation of the echo state network, a particle filter is exploited to recursively update the output weights when new condition monitoring data of the in-situ device becomes available. In this way, the degradation model is adaptive to varying external environments and operational modes. Another neural network method involving time-delayed neural network for the RUL prediction of IGBTs can be found in [114].

In [119], Gaussian processes regression is applied to the RUL prediction of IGBTs. For the degradation modeling, the nonlinear relationship between the decrement of on-state collector-emitter voltage $\Delta V_{\mathrm{ce}, \text { on }}$ and the condition monitoring

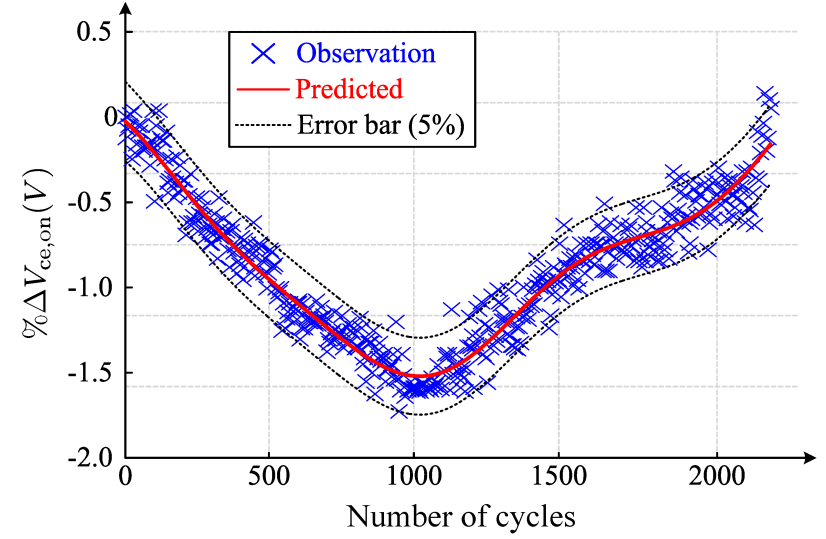

Fig. 21. Gaussian processes regression for the remaining useful life prediction of IGBTs [119].

time is established by the Gaussian processes regression. Since Gaussian process is formulated with the Bayesian framework, it is able to predict the uncertainty of variation $\Delta V_{\text {ce,on }}$ intrinsically. It can be seen from Fig. 21 that the error bar of the evolution of $\Delta V_{\text {ce,on }}$ is explicitly derived, which can be further utilized for the calculation of the confidence interval of RUL. Another example of kernel method for RUL prediction can be found in [74], where a support vector machine is applied to the degradation modeling of a buck converter.

To make AI-based methods of the RUL prediction more practical for field applications, more efforts should be devoted to the following aspects:

1) Uncertainty quantification: Compared to other regression-related tasks, e.g., control applications, the capability of uncertainty quantification is more critical for RUL prediction. As shown in Fig. 19, the RUL is a random variable and thus quantification of the confidence interval is essential for the optimal decision-making. These uncertainties come from the population heterogeneity, measurement noise, varying operational settings, etc, which should be comprehensively considered for a practical solution. AI methods are rather challenging for the uncertainty quantification of prediction results considering the black-box feature. Several feasible approaches include 
the Monte-Carlo methods [114], incorporating particle filter in the neural network [111], and Bayesian-based AI methods (e.g., Gaussian process, relevance vector machine). Another promising direction is the stochastic data-driven methods [154], [160], [168], which can intrinsically provide the PDF of the RUL for calculating the confidence interval.

2) Adaptive capability: It is concerned with the the model parameter tuning layer in Fig. 14 for connecting the offline models and the online models, which is a key step for practical applications. If a specific AI method lacks an adaptive capability, its application is limited since one prerequisite is that the training data and the test data should be generated under similar situations (e.g., external environments and operational modes) and share a high-level similarity [95]. It is challenging for power electronics since operational settings of the insitu system (i.e., the test data) are quite different from that of the training dataset, which is generally obtained with accelerated testing experiments. The majority of the research [74], [114], [119] assumes that the operational settings of the in-situ system are identical to the training dataset (e.g., accelerated aging experiments), which may not be the case in field applications. Thus, the adaptive capability of the AI-based RUL prediction method is critical to bridge academic research and industrial applications. Other promising directions of model parameter tuning include the explicit mapping relationship derivations [169] and transfer learning [170], [171] of degradation characteristics under various operational settings (temperature, voltage, humidity, etc.). This may, however, imply intensive investigations of system models.

\section{Outlook on AI for Power Electronic Systems}

From the algorithm perspective, it is necessary to investigate the features of AI when it applies to different life-cycle phases. By using a power converter system, three specific examples are applied to illustrate the requirements of AI methods for each life-cycle phase.

For the heatsink design of a converter system, a large number of decision variables, e.g., weight, volume, pattern, need to be determined, which is essentially an optimization task. The metaheuristic methods are applied to the optimization that involves an iterative trial-and-error procedure. Although the computational effort is intensive, the design task is typically performed offline. There is less requirement on the algorithm speed in this case. Although the metaheuristic method-based optimization does not ensure a global solution, the sub-optimal heatsink design is still superior and satisfactory in most cases. Thus, the algorithm accuracy is not critical as well. The training dataset and interpretability of the optimization process are not required.

For the intelligent controller of a converter system, the realtime control errors, e.g., the voltage error, the current error, need to be returned to the controller for the adaptive updating in an online mode. Thus, the requirements of algorithm speed and accuracy are the most critical. In addition, the controller
Table VI: Requirements of AI for exemplary applications in design, control, and maintenance. High: +++, moderate: ++, low: +

\begin{tabular}{|r|c|c|c|}
\hline Requirements & $\begin{array}{c}\text { Heatsink } \\
\text { design } \\
\text { (Design) }\end{array}$ & $\begin{array}{c}\text { Intelligent } \\
\text { controller } \\
\text { (Control) }\end{array}$ & $\begin{array}{c}\text { RUL } \\
\text { prediction } \\
\text { (Maintenance) }\end{array}$ \\
\hline Computation Effort & +++ & ++ & ++ \\
\hline Algorithm Speed & + & +++ & ++ \\
\hline Accuracy & ++ & +++ & +++ \\
\hline Dataset requirement & + & + & +++ \\
\hline Interpretability & + & +++ & +++ \\
\hline
\end{tabular}

stability needs to be theoretically ensured and thus the interpretability is critical. The intelligent controller is generally tuned online, it is unnecessary to prepare the dataset for the model training.

For the RUL prediction of switching devices in a converter system, the requirement of the algorithm speed is moderate since the device degradation is slow and the long time span of decision making is acceptable. The degradation model for the RUL prediction can be prepared in offline mode and efficiently tuned in online mode, and the computational effort in this application is moderate. Since the model accuracy is highly dependent on the dataset, the dataset requirement, e.g., dataset quality, dataset size, label balance (e.g., limited abnormal data in the training dataset), etc., is the most critical. Moreover, the interpretability of the RUL prediction results with uncertainty is critical as well. As a result, a comparison of AI algorithms in each phase of the life-cycle of power electronic systems is provided in Table VI.

It is concluded that AI possesses immense potentialities in power electronic systems. Many opportunities and issues are yet to be explored as follows:

1) Motivations and Justifications of AI Applied to Power Electronic Systems: Although there are numerous studies on AI for power electronic systems in the literature since the 1990s, the practical implementations in industry are still limited, which is a sharp contrast compared to the claimed AI potentials. It is necessary for deeper investigations into tasks where AI can essentially outperform conventional methods. The justifications of AI-based solutions should be clearly identified by comparing to conventional methods from the industrial perspectives, e.g., implementation complexity, algorithm accuracy and robustness, algorithm accountability, extra hardware cost, computational energy consumption, embedded capability, etc.

2) Interwoven AI implementations through Life-cycle Phases: Implementations of AI in each life-cycle phase of design, control, and maintenance, will facilitate flexible functional interactions. This feature is beneficial to overall performance optimization and procedure simplification. It enables the system capability in managing data flow between electrical and other disciplines (e.g., mechanical area) [13] as well. For example, aging information obtained by the AI-based system parameter identification can be flexibly incorporated into the AIbased controller for reliability improvement. Therefore, more attention should be paid to the interwoven inter- 
actions powered by AI.

3) Multilevel Information Fusion: Robustness is essential for safety-critical power electronic systems. Multiple sources of information and models are available in most cases for a specific application of power electronic systems. If these information sources and models are simultaneously exploited, possible biases can be mitigated to improve robustness. Multilevel information fusion can be performed at the data-level [160], [172], feature-level, decision-level [96], and their combinations, in order to exploit the insights of each information sources. For example, the well-established differential equations of power converter system can be integrated with $\mathrm{AI}$ as a hybrid solution for condition monitoring. As a result, the advantages from both the model-driven side and the data-driven side can be gained for better accuracy and robustness.

4) Computation-light AI: Compared to other industrial areas (e.g., image recognition), one of the key features of power electronic systems is that there is no powerful computation unit. While real-time applications, e.g., control, impose a rigid requirement on the algorithm speed. Although complex deep learning techniques [170] can provide superior performance, it is computationally intensive for power electronic systems. A prospective direction is the computation-light AI algorithms that can be implemented on cost-effective units but provide comparable performance with deep learning algorithms.

5) Data-light AI: One of the bottlenecks of AI implementation on power electronic systems is the dataset. For example, AI-based solutions for remaining useful life prediction requires the dataset to be versatile enough for accurate degradation behavior learning. However, the dataset size is generally small since the degradation experiments are resource-consuming. This situation is even worse for safety-critical cases. Thus, developing AI algorithms with lower dataset requirement, i.e., datalight AI solutions that can provide acceptable performance in the presence of poor datasets, is a prospective direction.

6) Explainable AI: Most of the AI algorithms in power electronics suffer from the "black-box" feature. For example, most of the AI-based solutions for remaining useful life prediction can only provide a point estimation without sensitivity analysis and uncertainty quantification. It makes AI-based solutions opaque and less convincing for practitioners to implement in industry applications, especially for safety-critical cases. There is a pressing need to improve the algorithm transparency for explainable AI with better interpretability. Understanding how models come up the decisions is critical for model simplification and safety, with which AI solutions can be implemented with confidence.

7) Dataset Privacy: An increasing attention has been paid to the data privacy, e.g., General Data Protection Regulation (GDPR) [173] in the European Union. With these critical regulations, the training of standard AI algorithms is challenging since a centralized data collec- tion may be not feasible in the future. Thus, for power electronics applications, it is promising to develop a collaborative learning scheme for AI algorithms without collectively aggregating data from different locations, e.g., federated learning [174]. It is well aligned with the trend of data privacy regulations for the implementation of AI solutions.

8) Power Electronics Database: Due to the complexity of system dynamics of power electronics, extensive datasets are required for the model training, especially for the maintenance applications. While the experimental testing for data collection is generally timeconsuming and expensive. There is a compelling demand for building up common power electronics data and knowledge base. These open-source datasets are critical to benchmark algorithm performance and accelerating application development. It will benefit the global power electronics communities in academia and industry.

\section{CONCLUSIONS}

Existing AI methods in power electronic systems are comprehensively reviewed in this paper. New findings are identified as

1) From the application perspective, the AI methods applied in power electronic systems can be categorized as the design, control, and maintenance. The usage percentage, application trend, features, and requirements of AI in each life-cycle phase are discussed.

2) From the method perspective, the AI methods applied in power electronic systems can be categorized as expert system, fuzzy logic, metaheuristic methods, and machine learning. The usage percentage, advantages, and limitations of relevant AI algorithms in each category are comprehensively compared.

3) From the function perspective, the AI-related applications are essentially dealing with the optimization, classification, regression, and data structure exploration.

4) The milestones of relevant algorithm variants and applications are identified and organized as a timeline map.

5) For each life-cycle phase, illustrative examples are discussed and the challenges and future research opportunities are identified.

\section{REFERENCES}

[1] C. M. Bishop, Pattern Recognition and Machine Learning. Springer, 2006.

[2] I. Goodfellow, Y. Bengio, and A. Courville, Deep Learning. MIT press, 2016.

[3] T. Wu, Z. Wang, B. Ozpineci, M. Chinthavali, and S. Campbell, "Automated heatsink optimization for air-cooled power semiconductor modules," IEEE Trans. Power Electron., vol. 34, no. 6, pp. 5027-5031, Jun. 2019.

[4] X. Zhan, W. Wang, and H. Chung, "A neural-network-based color control method for multi-color LED systems," IEEE Trans. Power Electron., vol. 34, no. 8, pp. 7900-7913, Aug. 2019.

[5] C. Wei, Z. Zhang, W. Qiao, and L. Qu, "Reinforcement-learning-based intelligent maximum power point tracking control for wind energy conversion systems," IEEE Trans. Ind. Electron., vol. 62, no. 10, pp. 6360-6370, Oct. 2015. 
[6] C. Wei, Z. Zhang, W. Qiao, and L. Y. Qu, "An adaptive networkbased reinforcement learning method for MPPT control of PMSG wind energy conversion systems," IEEE Trans. Power Electron., vol. 31 , no. 11, pp. 7837-7848, Nov. 2016.

[7] I. Bandyopadhyay, P. Purkait, and C. Koley, "Performance of a classifier based on time-domain features for incipient fault detection in inverter drives," IEEE Trans. Ind. Informat., vol. 15, no. 1, pp. 3-14, Jan. 2019.

[8] A. E. Mejdoubi, H. Chaoui, J. Sabor, and H. Gualous, "Remaining useful life prognosis of supercapacitors under temperature and voltage aging conditions," IEEE Trans. Ind. Electron., vol. 65, no. 5, pp. $4357-$ 4367, May 2018.

[9] F. Tao, H. Zhan, A. Liu, and A. Y. C. Nee, "Digital twin in industry: State-of-the-art," IEEE Trans. Ind. Informat., vol. 15, no. 4, pp. 24052415, Apr. 2019.

[10] X. He, W. Shi, W. Li, H. Luo, and R. Zhao, "Reliability enhancement of power electronics systems by big data science," Proc. CSEE, vol. 37, no. 1, pp. 209-221, Jan. 2017.

[11] K. L. Tsui, Y. Zhao, and D. Wang, "Big data opportunities: system health monitoring and management," IEEE Access, vol. 7, pp. 68853 68 867, May 2019.

[12] S. E. De Leon-Aldaco, H. Calleja, and J. A. Alquicira, "Metaheuristic optimization methods applied to power converters: A review," IEEE Trans. Power Electron., vol. 30, no. 12, pp. 6791-6803, Dec. 2015.

[13] M. R. G. Meireles, P. E. M. Almeida, and M. G. Simoes, "A comprehensive review for industrial applicability of artificial neural networks," IEEE Trans. Ind. Electron., vol. 50, no. 3, pp. 585-601, Jun. 2003.

[14] B. K. Bose, "Neural network applications in power electronics and motor drives-an introduction and perspective," IEEE Trans. Ind. Electron., vol. 54, no. 1, pp. 14-33, Feb. 2007.

[15] B. K. Bose, "Artificial intelligence techniques in smart grid and renewable energy systems-some example applications," Proc. IEEE, vol. 105 , no. 11 , pp. 2262-2273, Nov. 2017.

[16] M. Seyedmahmoudian, B. Horan, T. K. Soon, R. Rahmani, A. M. Than Oo, S. Mekhilef, and A. Stojcevski, "State of the art artificial intelligence-based MPPT techniques for mitigating partial shading effects on PV systems-a review," Renewable Sustainable Energy Rev., vol. 64, pp. 435-455, Oct. 2016.

[17] A. Mellit and S. A. Kalogirou, "Artificial intelligence techniques for photovoltaic applications: A review," Progress Energy Combustion Sci., vol. 34, no. 5, pp. 574-632, Oct. 2008.

[18] H. S.-H. Chung, H. Wang, F. Blaabjerg, and M. Pecht, Reliability of Power Electronic Converter Systems. Institution Eng. Technol., 2015.

[19] M. Riera-Guasp, J. A. Antonino-Daviu, and G. Capolino, "Advances in electrical machine, power electronic, and drive condition monitoring and fault detection: State of the art," IEEE Trans. Ind. Electron., vol. 62, no. 3, pp. 1746-1759, Mar. 2015.

[20] H. Soliman, H. Wang, and F. Blaabjerg, "A review of the condition monitoring of capacitors in power electronic converters," IEEE Trans. Ind. Appl., vol. 52, no. 6, pp. 4976-4989, Nov./Dec. 2016.

[21] M. Pecht and R. Jaai, "A prognostics and health management roadmap for information and electronics-rich systems," Microelectronics Rel., vol. 50, no. 3, pp. 317-323, Mar. 2010.

[22] L. Duchesne, E. Karangelos, and L. Wehenkel, "Recent developments in machine learning for energy systems reliability management," Proc. IEEE, 2020, Early access.

[23] R. C. G. Joao Pinto, Burak Ozpineci, "Tutorial: Artificial intelligence applications to power electronics," in IEEE Energy Convers. Congr. Expo., 2019, pp. 1-139.

[24] J. Foutz, "Power supply circuit development estimating aid: an expert system application," in Annu. IEEE Applied Power Electron. Conf. and Expo., 1988, pp. 64-71.

[25] S. M. Chhaya and B. K. Bose, "Expert system aided automated design, simulation and controller tuning of AC drive system," in Proc. 21st Annu. Conf. IEEE Ind. Electron., vol. 1, 1995, pp. 712-718.

[26] W. Li and J. P. Ying, "Design and analysis artificial intelligence (AI) research for power supply - power electronics expert system (PEES)," in Annu. IEEE Applied Power Electron. Conf. and Expo., Vols 1-4, 2008, pp. 2009-2015.

[27] D. Fezzani, H. Piquet, and H. Foch, "Expert system for the CAD in power electronics - application to UPS," IEEE Trans. Power Electron., vol. 12, no. 3, pp. 578-586, May 1997.

[28] A. M. Elsaadawi, A. E. Kalas, and M. Fawzi, "Development of an expert system to fault diagnosis of three phase induction motor drive system," in Int. Middle-East Power System Conf., 2008, pp. 497-502.
[29] Y. Izuno, R. Takeda, and M. Nakaoka, "New fuzzy reasoning-based high-performance speed/position control schemes for ultrasonic motor driven by two-phase resonant inverter," in IEEE Industry Appl. Soc. Ann. Meeting, 1990, pp. 325-330.

[30] M. G. Simoes, B. K. Bose, and R. J. Spiegel, "Design and performance evaluation of a fuzzy-logic-based variable-speed wind generation system," IEEE Trans. Ind. Appl., vol. 33, no. 4, pp. 956-965, Jul./Aug. 1997.

[31] R. Osorio, J. M. Alonso, N. Vazquez, S. E. Pinto, F. D. Sorcia-Vazquez, M. Martinez, and L. M. Barrera, "Fuzzy logic control with an improved algorithm for integrated LED drivers," IEEE Trans. Ind. Electron., vol. 65 , no. 9, pp. 6994-7003, Sep. 2018.

[32] A. Bubshait and M. G. Simoes, "Design of fuzzy logic-based dynamic droop controller of wind turbine system for primary frequency support," in IEEE Industry Appl. Soc. Ann. Meeting, 2018, pp. 1-7.

[33] W. Q. Chen and A. M. Bazzi, "Logic-based methods for intelligent fault diagnosis and recovery in power electronics," IEEE Trans. Power Electron., vol. 32, no. 7, pp. 5573-5589, Jul. 2017.

[34] M. G. Simoes and A. Bubshait, "Frequency support of smart grid using fuzzy logic-based controller for wind energy systems," Energies, vol. 12, no. 8, pp. 1-15, Apr. 2019.

[35] B. N. Alajmi, K. H. Ahmed, S. J. Finney, and B. W. Williams, "Fuzzy-logic-control approach of a modified hill-climbing method for maximum power point in microgrid standalone photovoltaic system,' IEEE Trans. Power Electron., vol. 26, no. 4, pp. 1022-1030, Apr. 2011.

[36] C. L. Tseng, S. Y. Wang, S. C. Chien, and C. Y. Chang, "Development of a self-tuning TSK-Fuzzy speed control strategy for switched reluctance motor," IEEE Trans. Power Electron., vol. 27, no. 4, pp. 2141-2152, Apr. 2012.

[37] R. C. Garcia, W. I. Suemitsu, and J. O. P. Pinto, "Takagi-Sugeno fuzzy model and control of a boost converter using Type-I internal model control," in 39th Annu. Conf. the IEEE Ind. Electron. Soc., 2013, pp. 3794-3799.

[38] F. Zidani, D. Diallo, M. E. H. Benbouzid, and R. Nait-Said, "A fuzzybased approach for the diagnosis of fault modes in a voltage-fed PWM inverter induction motor drive," IEEE Trans. Ind. Electron., vol. 55, no. 2, pp. 586-593, Feb. 2008.

[39] R. Fullér, Introduction to Neuro-Fuzzy Systems. Springer Science \& Business Media, 2000, vol. 2

[40] Matlab, "Manual of Neural Network Toolbox," http: //matlab.izmiran.ru/help/toolbox/nnet/, 2005.

[41] J. Zhang, H. S. H. Chung, W. L. Lo, S. Y. Hui, and A. K. M. Wu, "Implementation of a decoupled optimization technique for design of switching regulators using genetic algorithms," IEEE Trans. Power Electron., vol. 16, no. 6, pp. 752-763, Nov. 2001.

[42] L. L. Jiang, D. L. Maskell, and J. C. Patra, "A novel ant colony optimization-based maximum power point tracking for photovoltaic systems under partially shaded conditions," Energy and Buildings, vol. 58, pp. 227-236, Mar. 2013.

[43] Q. Wang and S. Niu, "Design, modeling, and control of a novel hybridexcited flux-bidirectional-modulated generator-based wind power generation system," IEEE Trans. Power Electron., vol. 33, no. 4, pp. 30863096, Apr. 2018.

[44] S. Lyden and M. E. Haque, "A simulated annealing global maximum power point tracking approach for pv modules under partial shading conditions," IEEE Trans. Power Electron., vol. 31, no. 6, pp. 41714181, Jun. 2016.

[45] J. Zhang, Y. Shi, and Z.-H. Zhan, "Power electronic circuits design: A particle swarm optimization approach," in Asia-Pacific Conf. Simulated Evolution Learning, 2008, pp. 605-614.

[46] Y. C. Hung, F. J. Lin, J. C. Hwang, J. K. Chang, and K. C. Ruan, "Wavelet fuzzy neural network with asymmetric membership function controller for electric power steering system via improved differential evolution," IEEE Trans. Power Electron., vol. 30, no. 4, pp. 2350-2362, Apr. 2015.

[47] J. Yuan, B. Chen, B. Rao, C. Tian, W. Wang, and X. Xu, "Possible analogy between the optimal digital pulse width modulation technology and the equivalent optimisation problem," IET Power Electron., vol. 5 , no. 7, pp. 1026-1033, Aug. 2012.

[48] S. Singh and B. Singh, "Optimized passive filter design using modified particle swarm optimization algorithm for a 12-pulse converter-fed LCI-synchronous motor drive," IEEE Trans. Ind. Appl., vol. 50, no. 4, pp. 2681-2689, Jul./Aug. 2014

[49] B. Zhao, X. Zhang, and J. J. Huang, "AI algorithm-based twostage optimal design methodology of high-efficiency CLLC resonant converters for the hybrid AC-DC microgrid applications," IEEE Trans. Ind. Electron., vol. 66, no. 12, pp. 9756-9767, Dec. 2019. 
[50] F. J. Lin, L. T. Teng, and M. H. Yu, "Radial basis function network control with improved particle swarm optimization for induction generator system," IEEE Trans. Power Electron., vol. 23, no. 4, pp. 2157-2169, Jul. 2008.

[51] W. Wang, H. S. Chung, R. Cheng, C. S. Leung, X. Zhan, A. W. Lo, J. Kwok, C. J. Xue, and J. Zhang, "Training neural-networkbased controller on distributed machine learning platform for power electronics systems," in IEEE Energy Conversion Congr. Expo., 2017, pp. 3083-3089.

[52] W. M. Lin and C. M. Hong, "A new Elman neural network-based control algorithm for adjustable-pitch variable-speed wind-energy conversion systems," IEEE Trans. Power Electron., vol. 26, no. 2, pp. 473-481, Feb. 2011

[53] F. J. Lin, L. T. Teng, and H. Chu, "A robust recurrent wavelet neural network controller with improved particle swarm optimization for linear synchronous motor drive," IEEE Trans. Power Electron., vol. 23 , no. 6, pp. 3067-3078, Nov. 2008.

[54] M. A. Hassan and M. A. Abido, "Optimal design of microgrids in autonomous and grid-connected modes using particle swarm optimization," IEEE Trans. Power Electron., vol. 26, no. 3, pp. 755-769, Mar. 2011.

[55] K. Ishaque, Z. Salam, M. Amjad, and S. Mekhilef, "An improved particle swarm optimization (PSO)-based MPPT for PV with reduced steady-state oscillation," IEEE Trans. Power Electron., vol. 27, no. 8, pp. 3627-3638, Aug. 2012.

[56] H. Taghizadeh and M. T. Hagh, "Harmonic elimination of cascade multilevel inverters with nonequal DC sources using particle swarm optimization," IEEE Trans. Ind. Electron., vol. 57, no. 11, pp. 36783684, Nov. 2010.

[57] W. Wang, A. C. Liu, H. S. Chung, R. W. Lau, J. Zhang, and A. W. Lo, "Fault diagnosis of photovoltaic panels using dynamic current-voltage characteristics," IEEE Trans. Power Electron., vol. 31, no. 2, pp. 15881599, Feb. 2016

[58] Z. H. Liu, H. L. Wei, Q. C. Zhong, K. Liu, X. S. Xiao, and L. H. Wu, "Parameter estimation for VSI-fed PMSM based on a dynamic PSO with learning strategies," IEEE Trans. Power Electron., vol. 32, no. 4, pp. 3154-3165, Apr. 2017

[59] M. Liserre, A. Dell'Aquila, and F. Blaabjerg, "Genetic algorithmbased design of the active damping for an LCL-filter three-phase active rectifier," IEEE Trans. Power Electron., vol. 19, no. 1, pp. 76-86, Jan. 2004.

[60] B. Ji, X. G. Song, E. Sciberras, W. P. Cao, Y. H. Hu, and V. Pickert, "Multiobjective design optimization of IGBT power modules considering power cycling and thermal cycling," IEEE Trans. Power Electron. vol. 30 , no. 5, pp. 2493-2504, May 2015 .

[61] F. Wang, G. Chen, D. Boroyevich, S. Ragon, M. Arpilliere, and V. R. Stefanovic, "Analysis and design optimization of diode frontend rectifier passive components for voltage source inverters," IEEE Trans. Power Electron., vol. 23, no. 5, pp. 2278-2289, Sep. 2008.

[62] M. D'Antonio, C. Shi, B. Wu, and A. Khaligh, "Design and optimization of a solar power conversion system for space applications," IEEE Trans. Ind. Appl., vol. 55, no. 3, pp. 2310-2319, May/Jun. 2019.

[63] B. Chen, X. Liang, and N. Wan, "Design methodology for inductorintegrated Litz-wired high-power medium-frequency transformer with the nanocrystalline core material for isolated DC-link stage of solidstate transformer," IEEE Trans. Power Electron., 2020, Early access.

[64] L. G. Junior, J. O. P. Pinto, J. A. B. Filho, and G. Lambert-Torres, "Recursive least square and genetic algorithm based tool for PID controllers tuning," in Int. Conf. Intelligent Sys. Appl. to Power Syst., 2007, pp. 1-6.

[65] M. J. Schutten and D. A. Torrey, "Genetic algorithms for control of power converters," in 26th Annu. IEEE Power Electron. Specialists Conf., Vols I and II, 1995, pp. 1321-1326.

[66] M. S. A. Dahidah and V. G. Agelidis, "Selective harmonic elimination PWM control for cascaded multilevel voltage source converters: A generalized formula," IEEE Trans. Power Electron., vol. 23, no. 4, pp. 1620-1630, Jul. 2008.

[67] M. J. Neath, A. K. Swain, U. K. Madawala, and D. J. Thrimawithana, "An optimal PID controller for a bidirectional inductive power transfer system using multiobjective genetic algorithm," IEEE Trans. Power Electron., vol. 29, no. 3, pp. 1523-1531, Mar. 2014.

[68] A. Mehrizi-Sani and S. Filizadeh, "An optimized space vector modulation sequence for improved harmonic performance," IEEE Trans. Ind. Electron., vol. 56, no. 8, pp. 2894-2903, Aug. 2009.

[69] M. Garaj, K. Y. Hong, H. S.-H. Chung, J. Zhou, and A. W.-L. Lo, "Photovoltaic panel health diagnostic system for solar power plants," in IEEE Energy Conversion Congr. Expo., 2018, pp. 1-6.
[70] J. Zhang, H. S. H. Chung, A. W. L. Lo, and T. Huang, "Extended ant colony optimization algorithm for power electronic circuit design," IEEE Trans. Power Electron., vol. 24, no. 1-2, pp. 147-162, Jan. 2009.

[71] F. J. Lin, P. K. Huang, H. C. Wang, and L. T. Teng, "An induction generator system using fuzzy modeling and recurrent fuzzy neural network," IEEE Trans. Power Electron., vol. 22, no. 1, pp. 260-271, Jan. 2007.

[72] B. X. Li and K. S. Low, "Low sampling rate online parameters monitoring of DC-DC converters for predictive-maintenance using biogeography-based optimization," IEEE Trans. Power Electron. vol. 31, no. 4, pp. 2870-2879, Apr. 2016.

[73] Q. Sun, Y. Wang, Y. Jiang, and L. Shao, "Non-invasive condition monitoring for boost converter based on crow search algorithm," $J$. Intell. \& Fuzzy Syst., vol. 34, no. 6, pp. 3661-3670, Jun. 2018.

[74] L. Wang, J. Yue, Y. Su, F. Lu, and Q. Sun, "A novel remaining useful life prediction approach for superbuck converter circuits based on modified grey wolf optimizer-support vector regression," Energies, vol. 10, no. 4, p. 459, Apr. 2017.

[75] A. Kavousi, B. Vahidi, R. Salehi, M. K. Bakhshizadeh, N. Farokhnia, and S. H. Fathi, "Application of the bee algorithm for selective harmonic elimination strategy in multilevel inverters," IEEE Trans. Power Electron., vol. 27, no. 4, pp. 1689-1696, Apr. 2012.

[76] M. H. Etesami, N. Farokhnia, and S. Hamid Fathi, "Colonial competitive algorithm development toward harmonic minimization in multilevel inverters," IEEE Trans. Ind. Informat., vol. 11, no. 2, pp. 459-466, Apr. 2015.

[77] K. Haghdar, "Optimal DC source influence on selective harmonic elimination in multilevel inverters using teaching-learning-based optimization," IEEE Trans. Ind. Electron., vol. 67, no. 2, pp. 942-949, Feb. 2020.

[78] J. Zhang, Z. H. Zhan, Y. Lin, N. Chen, Y. J. Gong, J. H. Zhong, H. S. H Chung, Y. Li, and Y. H. Shi, "Evolutionary computation meets machine learning: A survey," IEEE Computational Intell. Mag., vol. 6, no. 4, pp. 68-75, Nov. 2011.

[79] D. Chiozzi, M. Bernardoni, N. Delmonte, and P. Cova, "A neural network based approach to simulate electrothermal device interaction in SPICE environment," IEEE Trans. Power Electron., vol. 34, no. 5, pp. 4703-4710, May 2019.

[80] T. Dragicevic, P. Wheeler, and F. Blaabjerg, "Artificial intelligence aided automated design for reliability of power electronic systems," IEEE Trans. Power Electron., vol. 34, no. 8, pp. 7161-7171, Aug. 2019.

[81] M. G. Simoes and B. K. Bose, "Neural-network-based estimation of feedback signals for a vector controlled induction-motor drive," IEEE Trans. Ind. Appl., vol. 31, no. 3, pp. 620-629, May/Jun. 1995.

[82] J. Zhao and B. K. Bose, "Neural-network-based waveform processing and delayless filtering in power electronics and AC drives," IEEE Trans. Ind. Electron., vol. 51, no. 5, pp. 981-991, Oct. 2004.

[83] S. K. Mondal, J. O. P. Pinto, and B. K. Bose, "A neural-network-based space-vector PWM controller for a three-level voltage-fed inverter induction motor drive," IEEE Trans. Ind. Appl., vol. 38, no. 3, pp. 660-669, May/Jun. 2002.

[84] C. M. Lin, K. N. Hung, and C. F. Hsu, "Adaptive neuro-wavelet control for switching power supplies," IEEE Trans. Power Electron., vol. 22, no. 1, pp. 87-95, Jan. 2007.

[85] M. Novak and T. Dragicevic, "Supervised imitation learning of finite set model predictive control systems for power electronics," IEEE Trans. Ind. Electron., 2020, Early access.

[86] H. Soliman, P. Davari, H. Wang, and F. Blaabjerg, "Capacitance estimation algorithm based on DC-link voltage harmonics using artificial neural network in three-phase motor drive systems," in IEEE Energy Conversion Congr. Expo., 2017, pp. 5795-5802.

[87] H. Soliman, H. Wang, B. Gadalla, and F. Blaabjerg, "Condition monitoring for DC-link capacitors based on artificial neural network algorithm," in IEEE 5th Int. Conf. Power Eng., Energy and Electr. Drives, 2015, pp. 587-591.

[88] H. Soliman, I. Abdelsalam, H. Wang, and F. Blaabjerg, "Artificial neural network based DC-link capacitance estimation in a diode-bridge front-end inverter system," in IEEE 3rd Int. Future Energy Electron. Conf. ECCE Asia, 2017, pp. 196-201.

[89] Z. J. Huang, Z. S. Wang, and H. G. Zhang, "Multilevel feature moving average ratio method for fault diagnosis of the microgrid inverter switch," IEEE-CAA J. of Automatica Sinica, vol. 4, no. 2, pp. 177185, Apr. 2017.

[90] Z. J. Huang, Z. S. Wang, and H. G. Zhang, "A diagnosis algorithm for multiple open-circuited faults of microgrid inverters based on main 
fault component analysis," IEEE Trans. Energy Conversion, vol. 33, no. 3, pp. 925-937, Sep. 2018.

[91] Z. J. Huang, Z. S. Wang, and H. G. Zhang, "Multiple open-circuit fault diagnosis based on multistate data processing and subsection fluctuation analysis for photovoltaic inverter," IEEE Trans. Instrum. Meas., vol. 67, no. 3, pp. 516-526, Mar. 2018.

[92] S. Qiang and Y. Li, "Motor inverter fault diagnosis using wavelets neural networks," in 2013 IEEE Int. Conf. on Syst., Man, and Cybernetics, 2013, pp. 3168-3173.

[93] S. Mohagheghi, R. G. Harley, T. G. Habetler, and D. Divan, "Condition monitoring of power electronic circuits using artificial neural networks," IEEE Trans. Power Electron., vol. 24, no. 10, pp. 2363 2367, Oct. 2009

[94] S. Khomfoi and L. M. Tolbert, "Fault diagnostic system for a multilevel inverter using a neural network," IEEE Trans. Power Electron., vol. 22, no. 3, pp. 1062-1069, May 2007.

[95] Y. Cui, J. Shi, and Z. Wang, "Quantum assimilation-based state-ofhealth assessment and remaining useful life estimation for electronic systems," IEEE Trans. Ind. Electron., vol. 63, no. 4, pp. 2379-2390, Apr. 2016.

[96] P. Tamilselvan, P. F. Wang, and M. Pecht, "A multi-attribute classification fusion system for insulated gate bipolar transistor diagnostics," Microelectronics Rel., vol. 53, no. 8, pp. 1117-1129, Aug. 2013.

[97] Z. S. Wang, Z. J. Huang, C. H. Song, and H. G. Zhang, "Multiscale adaptive fault diagnosis based on signal symmetry reconstitution preprocessing for microgrid inverter under changing load condition," IEEE Trans. Smart Grid, vol. 9, no. 2, pp. 797-806, Mar. 2018.

[98] D. Diallo, M. E. H. Benbouzid, D. Hamad, and X. Pierre, "Fault detection and diagnosis in an induction machine drive: A pattern recognition approach based on concordia stator mean current vector," IEEE Trans. Energy Conversion, vol. 20, no. 3, pp. 512-519, Sep. 2005.

[99] C. S. Chen, "TSK-Type self-organizing recurrent-neural-fuzzy control of linear microstepping motor drives," IEEE Trans. Power Electron., vol. 25 , no. 9, pp. 2253-2265, Sep. 2010.

[100] R. J. Wai and L. C. Shih, "Adaptive fuzzy-neural-network design for voltage tracking control of a DC-DC boost converter," IEEE Trans. Power Electron., vol. 27, no. 4, pp. 2104-2115, Apr. 2012.

[101] P. Z. Grabowski, M. P. Kazmierkowski, B. K. Bose, and F. Blaabjerg, "A simple direct-torque neuro-fuzzy control of PWM-inverter-fed induction motor drive," IEEE Trans. Ind. Electron., vol. 47, no. 4, pp. 863-870, Aug. 2000

[102] T. Kamel, Y. Biletskiy, and L. Chang, "Capacitor aging detection for the DC filters in the power electronic converters using ANFIS algorithm," in IEEE 28th Canadian Conf. Elect. Computer Eng., 2015, pp. 663668.

[103] A. Soualhi, M. Makdessi, R. German, F. R. Echeverria, H. Razik, A. Sari, P. Venet, and G. Clerc, "Heath monitoring of capacitors and supercapacitors using the neo-fuzzy neural approach," IEEE Trans. Ind. Informat., vol. 14, no. 1, pp. 24-34, Jan. 2018.

[104] C. Schauder, "Adaptive speed identification for vector control of induction-motors without rotational transducers," IEEE Trans. Ind. Appl., vol. 28, no. 5, pp. 1054-1061, Sep./Oct. 1992.

[105] L. E. B. da Silva, B. K. Bose, and J. O. P. Pinto, "Recurrent-neuralnetwork-based implementation of a programmable cascaded low-pass filter used in stator flux synthesis of vector-controlled induction motor drive," IEEE Trans. Ind. Electron., vol. 46, no. 3, pp. 662-665, Jun. 1999.

[106] X. G. Fu and S. H. Li, "Control of single-phase grid-connected converters with LCL filters using recurrent neural network and conventional control methods," IEEE Trans. Power Electron., vol. 31, no. 7, pp. 5354-5364, Jul. 2016

[107] J. O. P. Pinto, B. K. Bose, and L. E. B. da Silva, "A stator-flux-oriented vector-controlled induction motor drive with space-vector PWM and flux-vector synthesis by neural networks," IEEE Trans. Ind. Appl., vol. 37, no. 5, pp. 1308-1318, Sep./Oct. 2001.

[108] P. Xiao, G. K. Venayagamoorthy, K. A. Corzine, and J. Huang, "Recurrent neural networks based impedance measurement technique for power electronic systems," IEEE Trans. Power Electron., vol. 25, no. 2, pp. 382-390, Feb. 2010.

[109] Y. Zhang, Z. Wang, H. Wang, and F. Blaabjerg, "Artificial intelligenceaided thermal model considering cross-coupling effects," IEEE Trans. Power Electron., 2020, Early access.

[110] M. R. Habibi, H. R. Baghaee, T. Dragicevic, and F. Blaabjerg, "Detection of false data injection cyber-attacks in DC microgrids based on recurrent neural networks," IEEE J. Emerging Selected Topics Power Electron., 2020, Early access.
[111] Z. Li, Z. Zheng, and R. Outbib, "A prognostic methodology for power MOSFETs under thermal stress using echo state network and particle filter," Microelectronics Rel., vol. 88-90, pp. 350-354, Sep. 2018.

[112] Z. J. Huang, Z. S. Wang, X. S. Yao, and H. G. Zhang, "Multi-switches fault diagnosis based on small low-frequency data for voltage-source inverters of PMSM drives," IEEE Trans. Power Electron., vol. 34, no. 7, pp. 6845-6857, Jul. 2019

[113] Z. Y. Xue, K. S. Xiahou, M. S. Li, T. Y. Ji, and Q. H. Wu, "Diagnosis of multiple open-circuit switch faults based on long short-term memory network for DFIG-based wind turbine systems," IEEE J. Emerg. Sel. Topics Power Electron., 2019, Early access.

[114] A. Alghassi, S. Perinpanayagam, and M. Samie, "Stochastic RUL calculation enhanced with TDNN-based IGBT failure modeling," IEEE Trans. Rel., vol. 65, no. 2, pp. 558-573, Jun. 2016.

[115] S. Kiranyaz, A. Gastli, L. Ben-Brahim, N. Al-Emadi, and M. Gabbouj, "Real-time fault detection and identification for MMC using 1-D convolutional neural networks," IEEE Trans. Ind. Electron., vol. 66 , no. 11, pp. 8760-8771, Nov. 2019.

[116] C. Y. Yin, H. Lu, M. Musallam, C. Bailey, and C. M. Johnson, "A prognostic assessment method for power electronics modules," in 2nd Electron. Syst.-Integration Technol. Conf., 2008, pp. 1353-1358.

[117] B. Cai, Y. Zhao, H. Liu, and M. Xie, "A data-driven fault diagnosis methodology in three-phase inverters for PMSM drive systems," IEEE Trans. Power Electron., vol. 32, no. 7, pp. 5590-5600, Jul. 2017.

[118] S. S. Moosavi, A. Kazemi, and H. Akbari, "A comparison of various open-circuit fault detection methods in the IGBT-based DC/AC inverter used in electric vehicle," Eng. Failure Analysis, vol. 96, pp. 223-235, Feb. 2019.

[119] S. H. Ali, M. Heydarzadeh, S. Dusmez, X. Li, A. S. Kamath, and B. Akin, "Lifetime estimation of discrete IGBT devices based on gaussian process," IEEE Trans. Ind. Appl., vol. 54, no. 1, pp. 395403, Jan./Feb. 2018.

[120] J. R. Celaya, A. Saxena, S. Saha, and K. Goebel, "Prognostics of power MOSFETs under thermal stress accelerated aging using data-driven and model-based methodologies," in Annu. Conf.Prognostics Health Manage. Soc., 2011, pp. 1-10.

[121] A. Lidozzi, L. Solero, F. Crescimbini, and A. Di Napoli, "SVM PMSM drive with low resolution hall-effect sensors," IEEE Trans. Power Electron., vol. 22, no. 1, pp. 282-290, Jan. 2007

[122] N. A. Ahmed and A. K. Al-Othman, "Photovoltaic system with voltagebased maximum power point tracking using support vector machine," in Proc. 5th IEEE Conf. Ind. Electron. Appl., Vol 4, 2010, pp. 539-544.

[123] F. Mei, N. Liu, H. Y. Miao, Y. Pan, H. Y. Sha, and J. Y. Zheng, "Online fault diagnosis model for locomotive traction inverter based on wavelet transform and support vector machine," Microelectronics Rel., vol. 88-90, pp. 1274-1280, Sep. 2018.

[124] C. Delpha, H. Chen, and D. Diallo, "SVM based diagnosis of inverter fed induction machine drive: A new challenge," in 38th Ann. Conf. IEEE Ind. Electron. Soc., 2012, pp. 3931-3936.

[125] X. X. Zheng and P. Peng, "Fault diagnosis of wind power converters based on compressed sensing theory and weight constrained AdaBoostSVM," J. Power Electron., vol. 19, no. 2, pp. 443-453, Mar. 2019.

[126] A. G. Abo-Khalil and D. Lee, "DC-Link capacitance estimation in AC/DC/AC PWM converters using voltage injection," IEEE Trans. Ind. Appl., vol. 44, no. 5, pp. 1631-1637, Sep./Oct. 2008.

[127] T. Z. Wang, H. Xu, J. G. Han, E. Elbouchikhi, and M. E. H. Benbouzid, "Cascaded H-Bridge multilevel inverter system fault diagnosis using a PCA and multiclass relevance vector machine approach," IEEE Trans. Power Electron., vol. 30, no. 12, pp. 7006-7018, Dec. 2015.

[128] S. Q. Zhou, L. W. Zhou, and P. J. Sun, "Monitoring potential defects in an IGBT module based on dynamic changes of the gate current," IEEE Trans. Power Electron., vol. 28, no. 3, pp. 1479-1487, Mar. 2013

[129] J. Zhang, H. S. Chung, and W. Lo, "Clustering-based adaptive crossover and mutation probabilities for genetic algorithms," IEEE Trans. on Evolutionary Computation, vol. 11, no. 3, pp. 326-335, 2007.

[130] E. G. Strangas, S. Aviyente, and S. S. H. Zaidi, "Time-frequency analysis for efficient fault diagnosis and failure prognosis for interior permanent-magnet AC motors," IEEE Trans. Ind. Electron., vol. 55, no. 12, pp. 4191-4199, Dec. 2008.

[131] S. Zhao, V. Makis, S. Chen, and Y. Li, "Health assessment method for electronic components subject to condition monitoring and hard failure," IEEE Trans. Instrum. Meas., vol. 68, no. 1, pp. 138-150, Jan. 2019.

[132] Y. Z. Lu and A. Christou, "Prognostics of IGBT modules based on the approach of particle filtering," Microelectronics Rel., vol. 92, pp. 96-105, Jan. 2019. 
[133] M. Rigamonti, P. Baraldi, A. Alessi, E. Zio, D. Astigarraga, and A. Galarza, "An ensemble of component-based and population-based self-organizing maps for the identification of the degradation state of insulated-gate bipolar transistors," IEEE Trans. Rel., vol. 67, no. 3, pp. 1304-1313, Sep. 2018.

[134] N. Femia, G. Spagnuolo, and V. Tucci, "State-space models and order reduction for dc-dc switching converters in discontinuous modes," IEEE Trans. Power Electron., vol. 10, no. 6, pp. 640-650, 1995.

[135] J. F. Martins, V. F. Pires, C. Lima, and A. J. Pires, "Fault detection and diagnosis of grid-connected power inverters using PCA and current mean value," in 38th Annu. Conf. IEEE Ind. Electron. Soc., 2012, pp. $5185-5190$.

[136] W. Chen, L. Zhang, K. Pattipati, A. M. Bazzi, S. Joshi, and E. M. Dede, "Data-driven approach for fault prognosis of SiC MOSFETs," IEEE Trans. Power Electron., vol. 35, no. 4, pp. 4048-4062, Apr. 2020.

[137] J. Han, M. Kamber, and J. Pei, Data Mining: concepts and techniques, 3rd ed. The Morgan Kaufmann, 2012.

[138] M. Glavic, R. Fonteneau, and D. Ernst, "Reinforcement learning for electric power system decision and control: Past considerations and perspectives," IFAC Papersonline, vol. 50, no. 1, pp. 6918-6927, Jul. 2017.

[139] R. S. Sutton and A. G. Barto, Reinforcement Learning: An Introduction. MIT press, 2018

[140] P. Kofinas, S. Doltsinis, A. I. Dounis, and G. A. Vouros, "A reinforcement learning approach for MPPT control method of photovoltaic sources," Renewable Energy, vol. 108, pp. 461-473, Aug. 2017.

[141] A. S. Elwer, S. A. Wahsh, M. O. Khalil, and A. M. Nur-Eldeen, "Intelligent fuzzy controller using particle swarm optimization for control of permanent magnet synchronous motor for electric vehicle," in Proc. 29th Annu. Conf. IEEE Ind. Electron. Soc., Vols 1 - 3,, 2003, pp. $1762-1766$.

[142] F. Harashima, Y. Demizu, S. Kondo, and H. Hashimoto, "Application of neutral networks to power converter control," in Conf. Record IEEE Industry Appl. Soc. Annu. Meeting,, 1989, pp. 1086-1091 vol.1.

[143] S. Khomfoi and L. M. Tolbert, "Fault diagnosis and reconfiguration for multilevel inverter drive using AI-based techniques," IEEE Trans. Ind. Electron., vol. 54, no. 6, pp. 2954-2968, Dec. 2007.

[144] H. Zhang, J. Zhao, R. Wang, and T. Ma, "Multi-objective reinforcement learning algorithm and its application in drive system," in Proc. 34th Annu. Conf. IEEE Ind. Electron. Soc., Vols 1-5,, 2008, pp. 225-230.

[145] G. Bramerdorfer, J. A. Tapia, J. J. Pyrhonen, and A. Cavagnino, "Modern electrical machine design optimization: Techniques, trends, and best practices," IEEE Trans. Ind. Electron., vol. 65, no. 10, pp. 7672-7684, Oct. 2018.

[146] C. Versele, O. Deblecker, and J. Lobry, "Multiobjective optimal choice and design of isolated dc-dc power converters," in Proc. 14th Eur. Conf. Power Electron. Appl., 2011, pp. 1-10.

[147] S. Vighetti, J. Ferrieux, and Y. Lembeye, "Optimization and design of a cascaded DC/DC converter devoted to grid-connected photovoltaic systems," IEEE Trans. Power Electron., vol. 27, no. 4, pp. 2018-2027, 2012.

[148] H. Helali, D. Bergogne, H. Morel, and J. B. H. Slama, "Power converter design methodology: Uses of multiple objective techniques for optimization of a $(42 / 14 \mathrm{~V})$ buck converter," in 4 th Int. Conf. Integrated Power Syst., 2006, pp. 1-5.

[149] H. Zhang, F. Mollet, C. Saudemont, and B. Robyns, "Experimental validation of energy storage system management strategies for a local DC distribution system of more electric aircraft," IEEE Trans. Ind. Electron., vol. 57, no. 12, pp. 3905-3916, Dec. 2010.

[150] Y. F. Yin, J. X. Liu, J. A. Sanchez, L. G. Wu, S. Vazquez, J. I. Leon, and L. G. Franquelo, "Observer-based adaptive sliding mode control of NPC converters: An RBF neural network approach," IEEE Trans. Power Electron., vol. 34, no. 4, pp. 3831-3841, Apr. 2019.

[151] F. J. T. Filho, L. M. Tolbert, and B. Ozpineci, "Real time selective harmonic minimization for multilevel inverters using genetic algorithm and artificial neural network angle generation," in Proc. 7th Int. Power Electron. Motion Control Conf., vol. 2, 2012, pp. 895-899.

[152] K. H. Tan, "Squirrel-cage induction generator system using wavelet petri fuzzy neural network control for wind power applications," IEEE Trans. Power Electron., vol. 31, no. 7, pp. 5242-5254, Jul. 2016.

[153] J. S. R. Jang, "ANFIS: adaptive-network-based fuzzy inference system," IEEE Trans. Syst., Man, and Cybernetics, vol. 23, no. 3, pp. 665-685, May/Jun. 1993.

[154] S. Zhao, V. Makis, S. Chen, and Y. Li, "Evaluation of reliability function and mean residual life for degrading systems subject to condition monitoring and random failure," IEEE Trans. Rel., vol. 67, no. 1 , pp. 13-25, Mar. 2018.
[155] S. Zhao, V. Makis, S. Chen, and Y. Li, "Health evaluation method for degrading systems subject to dependent competing risks," J. Syst. Eng. Electron., vol. 29, no. 2, pp. 436-444, Apr. 2018.

[156] IEEE, "IEEE standard framework for prognostics and health management of electronic systems," IEEE Std 1856-2017, pp. 1-31, 2017.

[157] S. Yang, D. Xiang, A. Bryant, P. Mawby, L. Ran, and P. Tavner, "Condition monitoring for device reliability in power electronic converters: A review," IEEE Trans. Power Electron., vol. 25, no. 11, pp. 2734 2752, Nov. 2010.

[158] H. Oh, B. Han, P. McCluskey, C. Han, and B. D. Youn, "Physics-offailure, condition monitoring, and prognostics of insulated gate bipolar transistor modules: A review," IEEE Trans. Power Electron., vol. 30, no. 5, pp. 2413-2426, May 2015

[159] M. Al-Greer, M. Armstrong, M. Ahmeid, and D. Giaouris, "Advances on system identification techniques for DC-DC switch mode power converter applications," IEEE Trans. Power Electron., vol. 34, no. 7, pp. 6973-6990, Jul. 2019.

[160] S. Zhao, S. Chen, F. Yang, E. Ugur, B. Akin, and H. Wang, "A composite failure precursor for condition monitoring and remaining useful life prediction of discrete power devices," IEEE Trans. Ind. Informat., 2020, Early access.

[161] Matlab, "Diagnostic Feature Designer," https://www.mathworks.com/ help/predmaint/gs/explore-features-in-diagnostic-featuredesigner.html, 2020.

[162] M. A. F. Pimentel, D. A. Clifton, L. Clifton, and L. Tarassenko, "A review of novelty detection," Signal Process., vol. 99, pp. 215-249, Jun. 2014

[163] R. Liu, B. Yang, E. Zio, and X. Chen, "Artificial intelligence for fault diagnosis of rotating machinery: A review," Mech. Syst. Signal Process., vol. 108, pp. 33 - 47, Aug. 2018.

[164] Matlab, "Predictive Maintenance Toolbox," https:// www.mathworks.com/products/predictive-maintenance.html, 2020.

[165] A. Hanif, Y. Yu, D. DeVoto, and F. Khan, "A comprehensive review toward the state-of-the-art in failure and lifetime predictions of power electronic devices," IEEE Trans. Power Electron., vol. 34, no. 5, pp. 4729-4746, May 2019.

[166] M. A. Eleffendi and C. M. Johnson, "In-service diagnostics for wirebond lift-off and solder fatigue of power semiconductor packages," IEEE Trans. Power Electron., vol. 32, no. 9, pp. 7187-7198, Sep. 2017.

[167] M.-F. Ng, J. Zhao, Q. Yan, G. J. Conduit, and Z. W. Seh, "Predicting the state of charge and health of batteries using data-driven machine learning," Nature Machine Intelligence, vol. 2, no. 3, pp. 161-170, Mar. 2020.

[168] X.-S. Si, W. Wang, C.-H. Hu, and D.-H. Zhou, "Remaining useful life estimation - a review on the statistical data driven approaches," Eur. $J$. Oper. Res., vol. 213, no. 1, pp. 1-14, Aug. 2011

[169] Alpha and Omega Semiconductor, "Power semiconductor reliability handbook," http://www.aosmd.com/media/reliability-handbook.pdf, Sunnyvale, CA, USA, pp. 8-10, May 2010.

[170] R. Zhao, R. Q. Yan, Z. H. Chen, K. Z. Mao, P. Wang, and R. X. Gao, "Deep learning and its applications to machine health monitoring," Mech. Syst. Signal Process., vol. 115, pp. 213-237, Jan. 2019.

[171] Q. Wang, G. Michau, and O. Fink, "Domain adaptive transfer learning for fault diagnosis," in Prognostics Syst. Health Manage. Conf., 2019, pp. 1-7.

[172] L. Liao, "Discovering prognostic features using genetic programming in remaining useful life prediction," IEEE Trans. Ind. Electron., vol. 61 , no. 5, pp. 2464-2472, May 2014.

[173] European Union, "General Data Protection Regulation (GDPR)," https: //gdpr.eu/, 2018.

[174] Q. Yang, Y. Liu, T. Chen, and Y. Tong, "Federated machine learning: Concept and applications," ACM Trans. Intell. Syst. Technol., vol. 10, no. 2, Jan. 2019. 


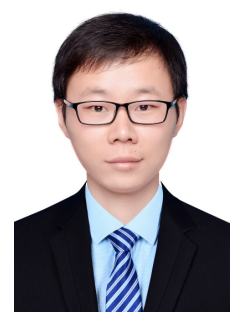

Shuai Zhao (S'14-M'18) received the B.E. (Hons), M.E., and Ph.D. degrees in information and communication engineering from Northwestern Polytechnical University, Xi' an, China, in 2011, 2014, and 2018 , respectively. He is currently a postdoctoral researcher with the Center of Reliable Power Electronics (CORPE), Department of Energy Technology, Aalborg University, Denmark. From Sep. 2014 to Sep. 2016, he was a visiting Ph.D. Student with the Department of Mechanical and Industrial Engineering at the University of Toronto, Toronto, ON, Canada, with the scholarship from China Scholarship Council (CSC). In August 2018, he was a visiting scholar with the Power Electronics and Drives Laboratory, Department of Electrical and Computer Science at the University of Texas at Dallas, Richardson, TX, USA. His research interests include system informatics, intelligent condition monitoring, diagnostics \& prognostics, and tailored AI tools for power electronic systems.

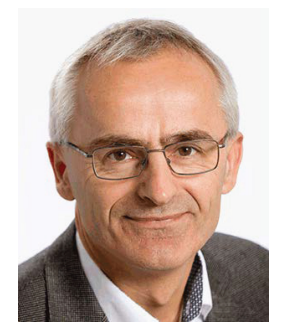

Frede Blaabjerg (S'86-M'88-SM'97-F'03) was with ABB-Scandia, Randers, Denmark, from 1987 to 1988. From 1988 to 1992, he got the PhD degree in Electrical Engineering at Aalborg University in 1995. He became an Assistant Professor in 1992, an Associate Professor in 1996, and a Full Professor of power electronics and drives in 1998. From 2017 he became a Villum Investigator. He is honoris causa at University Politehnica Timisoara (UPT), Romania and Tallinn Technical University (TTU) in Estonia.

His current research interests include power electronics and its applications such as in wind turbines, PV systems, reliability, harmonics and adjustable speed drives. He has published more than 600 journal papers in the fields of power electronics and its applications. He is the co-author of four monographs and editor of ten books in power electronics and its applications.

$\mathrm{He}$ has received 32 IEEE Prize Paper Awards, the IEEE PELS Distinguished Service Award in 2009, the EPE-PEMC Council Award in 2010, the IEEE William E. Newell Power Electronics Award 2014, the Villum Kann Rasmussen Research Award 2014, the Global Energy Prize in 2019, and the 2020 IEEE Edison Medal. He was the Editor-in-Chief of the IEEE Transactions on Power Electronics from 2006 to 2012. He has been Distinguished Lecturer for the IEEE Power Electronics Society from 2005 to 2007 and for the IEEE Industry Applications Society from 2010 to 2011 as well as 2017 to 2018. In 2019-2020 he serves a President of IEEE Power Electronics Society. He is Vice-President of the Danish Academy of Technical Sciences too. He is nominated in 2014-2019 by Thomson Reuters to be between the most 250 cited researchers in Engineering in the world.

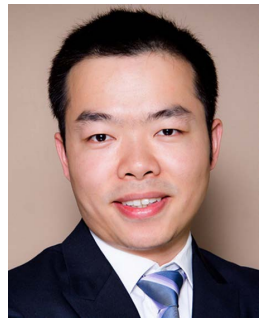

Huai Wang (M'12-SM'17) received the B.E. degree in electrical engineering, from Huazhong University of Science and Technology, Wuhan, China, in 2007 and the Ph.D. degree in power electronics, from the City University of Hong Kong, Hong Kong, in 2012. $\mathrm{He}$ is currently Professor with the Center of Reliable Power Electronics (CORPE), Department of Energy Technology at Aalborg University, Denmark. He was a Visiting Scientist with the ETH Zurich, Switzerland, from Aug. to Sep. 2014, and with the Massachusetts Institute of Technology (MIT), USA, from Sep. to Nov. 2013. He was with the ABB Corporate Research Center, Switzerland, in 2009. His research addresses the fundamental challenges in modelling and validation of power electronic component failure mechanisms, and application issues in system-level predictability, condition monitoring, circuit architecture, and robustness design. He leads a project on Light-AI for Cognitive Power Electronics.

Dr. Wang received the Richard M. Bass Outstanding Young Power Electronics Engineer Award from the IEEE Power Electronics Society in 2016, and the Green Talents Award from the German Federal Ministry of Education and Research in 2014. He is currently the Chair of IEEE PELS/IAS/IES Chapter in Denmark. He serves as an Associate Editor of IET Electronics Letters, IEEE JOURNAL OF EMERGING AND SELECTED TOPICS IN POWER ELECTRONICS, and IEEE TRANSACTIONS ON POWER ELECTRONICS. 